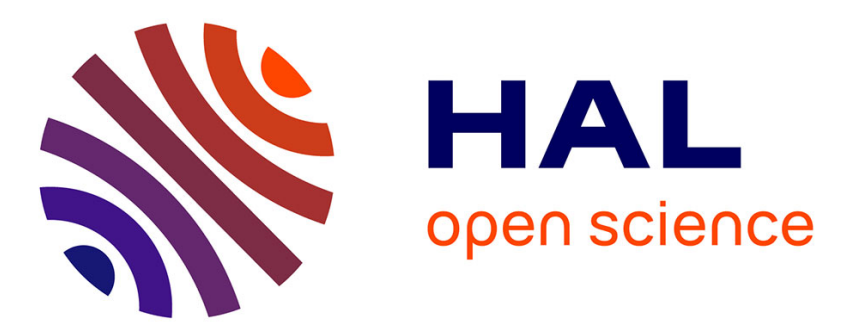

\title{
Symmetries of the electrodynamic interactions between chiral molecules
}

\author{
Richard Bonneville
}

\section{To cite this version:}

Richard Bonneville. Symmetries of the electrodynamic interactions between chiral molecules. Molecular Physics, 2008, 106 (21-23), pp.2627-2642. 10.1080/00268970802623802 . hal-00513241

\section{HAL Id: hal-00513241 \\ https://hal.science/hal-00513241}

Submitted on 1 Sep 2010

HAL is a multi-disciplinary open access archive for the deposit and dissemination of scientific research documents, whether they are published or not. The documents may come from teaching and research institutions in France or abroad, or from public or private research centers.
L'archive ouverte pluridisciplinaire HAL, est destinée au dépôt et à la diffusion de documents scientifiques de niveau recherche, publiés ou non, émanant des établissements d'enseignement et de recherche français ou étrangers, des laboratoires publics ou privés. 


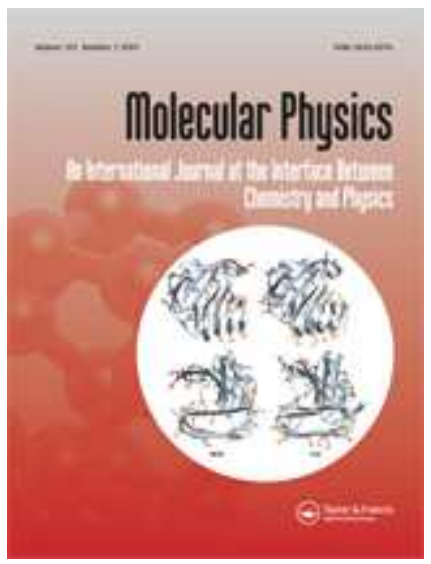

\section{Symmetries of the electrodynamic interactions between chiral molecules}

\begin{tabular}{|r|l|}
\hline Journal: & Molecular Physics \\
\hline Manuscript ID: & TMPH-2008-0271.R1 \\
\hline Manuscript Type: & Full Paper \\
\hline Date Submitted by the \\
Author: & 08-Nov-2008 \\
\hline Complete List of Authors: & BONNEVILLE, Richard; CNES, DSP/DA \\
\hline Keywords: & $\begin{array}{l}\text { electrodynamics, chirality, symmetries, liquid crystals, molecular } \\
\text { interactions }\end{array}$ \\
\hline
\end{tabular}

\section{s scholarONE" \\ Manuscript Central}




\begin{abstract}
A quantum electrodynamics calculation of the dynamic microscopic interactions between molecules beyond the electric dipole approximation is presented. The symmetry of the resulting terms is examined with respect to rotations, parity and index permutations. Their relative importance is also considered in the short distance limit. A dominant term which exists only in chiral molecules and which involves the coupling of the anisotropic parts of the molecular optical activities is evidenced. Associated with the coupling of the anisotropic parts of the molecular polarisabilities which is usually invoked to account for the isotropic nematic phase transition of liquid crystals, that term accounts for the possibility of the helical order which is observed in chiral nematics (cholesterics). Introducing in addition both the short range correlations due to the exclusion principle driven repulsive forces and the thermally induced effect of the static multipole interactions can explain the temperature dependence of the helical pitch. No assumption will be made upon the shape of the molecules.
\end{abstract}

\title{
Introduction
}

The intermolecular forces between 2 neutral molecules A and B include both static interactions between electric multipoles, due to the longitudinal electric field, and dynamic interactions due to the transverse electric and magnetic fields; in the quantum electrodynamics formulation of the field - matter interaction, those dynamic interactions are viewed as resulting from an exchange of virtual photons between A and B. The lowest order term of those dynamic interactions involves the exchange of 2 photons. If we limit ourselves to the electric dipole approximation of the photon - molecule coupling (the first term in a v/c expansion), we obtain the full expression of the Van der Waals forces including retardation effects, first derived by Casimir and Polder [1]. If we go beyond the electric dipole approximation $[2,3,4,5,6,7]$, new terms can be evidenced, among which some exist only in chiral molecules.

In the following section, we first recall how the quantum electrodynamics calculation of the microscopic interactions between molecules is performed in the electric dipole approximation as it will be our starting point. Then, in the next section, we perform the calculation of the terms beyond the electric dipole approximation involving magnetic dipole and electric quadrupole couplings, and magnetic quadrupole and electric octupole couplings. The symmetry of those terms will be carefully examined with respect to rotations, parity and index permutations by using the irreducible tensor formalism. Their relative importance will then be considered in the short distance limit where the retardation effects become negligible.

A dominant term which exists only in chiral molecules and which involves the coupling of the anisotropic parts of the molecular optical activities will be evidenced. It will be shown in the 
next section how that term, associated with the coupling of the anisotropic parts of the molecular polarisabilities which is usually invoked to account for the isotropic - nematic phase transition of liquid crystals, accounts for the possibility of the helical order which is observed in chiral nematics ("cholesterics").

However the resulting helical pitch does not depend on temperature. In the last section, we will see how introducing in addition both the short range correlations due to the exclusion principle driven repulsive forces and the thermally induced effects of the static multipole interactions can explain the temperature dependence of the helical pitch.

\section{Electric dipole approximation: Van der Waals forces}

In order to calculate the dynamic interaction between two molecules, we have used the elegant techniques of quantum electrodynamics $[8,9,10,11]$ instead of the original derivation by Casimir and Polder. By treating the field - matter coupling as a perturbation of the noninteracting molecules, the first non trivial, non vanishing term is the $4^{\text {th }}$ order one which describes the exchange of 2 photons. That term is the superposition of the 2 processes summarised by the 2 diagrams (i) and (ii) in fig. 1 . The total transition amplitude from the initial state $|i\rangle$ to the final state $|f\rangle$ is $T_{i \rightarrow f}=T_{i \rightarrow f}^{0}+T_{i \rightarrow f}^{\prime}+T^{\prime \prime}{ }_{i \rightarrow f}$ where $\mathrm{T}_{\mathrm{i} \rightarrow \mathrm{f}}^{0}=\exp \left[-\mathrm{iE}_{0}\left(\mathrm{t}_{\mathrm{f}}-\mathrm{t}_{\mathrm{i}}\right) \hbar^{-1}\right]$ accounts for the trivial, un-coupled case, and $\mathrm{T}_{\mathrm{i} \rightarrow \mathrm{f}}$ and $\mathrm{T}^{\prime \prime}{ }_{\mathrm{i} \rightarrow \mathrm{f}}$ respectively account for the diagrams (i) and (ii). In those expressions $E_{0}$ is the energy of the fundamental molecular state, and $t_{i}$ and $t_{f}$ are the initial and final times when the coupling is respectively switched on and off; actually $t_{i} \rightarrow-\infty$ and $t_{f} \rightarrow+\infty$.

In this first stage, the 4 interaction vortices are assumed to be of electric dipole type (E1). We hereunder briefly recall the derivation scheme of the effective interaction energy as it will subsequently be extended to higher order multipole interactions. A detailed calculation is given in Appendix A1.

$\mathcal{H}$ is the molecular Hamiltonian such that $\mathcal{H}|\mathrm{n}\rangle=\mathrm{E}_{\mathrm{n}}|\mathrm{n}\rangle$. The electromagnetic selfcouplings are handled through a re-normalisation of the molecular energy levels, which results in replacing $\mathrm{E}_{\mathrm{n}}$ by $\mathrm{E}_{\mathrm{n}}{ }^{*}=\mathrm{E}_{\mathrm{n}}+\Delta_{\mathrm{n}}(\mathrm{E}) \mp \mathrm{i} \Gamma_{\mathrm{n}}(\mathrm{E}) / 2$; for the fundamental level, $\Gamma_{0}(\mathrm{E})=0$. We will henceforth use the usual notations:

$\mathrm{P}_{\mathrm{m}}=|\mathrm{m}\rangle\langle\mathrm{m}|:$ projection operator onto the molecular states,

d, M, Q : respectively molecular electric dipole, magnetic dipole, electric quadrupole,

$\mathbf{k}, \mathbf{k}^{\prime}, \mathbf{e}, \mathbf{e}^{\prime}$ : wave-vectors and polarisations of the two virtual photons,

$\omega$ : photon frequency,

$\mathbf{R}_{\mathrm{AB}}$ : vector connecting the centres of two molecules,

$\mathrm{c}, \in_{0}, \hbar$ : speed of light, vacuum permittivity, Planck's constant.

The subscript A (respectively B) given to a physical quantity indicates that quantity refers to molecule A (respectively B).

Introducing the molecular polarisability tensor $\alpha_{\mathrm{kl}}(\omega)$ 


$$
\alpha_{\mathrm{kl}}(\omega)=\left(4 \pi \epsilon_{0}\right)^{-1} \sum_{\mathrm{m}} \frac{\left\langle 0\left|\mathrm{~d}_{\mathrm{k}} \mathrm{P}_{\mathrm{m}} \mathrm{d}_{1}\right| 0\right\rangle}{\mathrm{E}_{0}-\mathrm{E}_{\mathrm{m}}+\hbar \omega-\Delta_{\mathrm{m}}(\omega)+\frac{\mathrm{i} \Gamma_{\mathrm{m}}(\omega)}{2}}+\frac{\left\langle 0\left|\mathrm{~d}_{1} \mathrm{P}_{\mathrm{m}} \mathrm{d}_{\mathrm{k}}\right| 0\right\rangle}{\mathrm{E}_{0}-\mathrm{E}_{\mathrm{m}}-\hbar \omega-\Delta_{\mathrm{m}}(-\omega)-\frac{\mathrm{i} \Gamma_{\mathrm{m}}(-\omega)}{2}}
$$

and using the symmetry property $\alpha_{\mathrm{kl}}(-\omega)=\alpha_{\mathrm{lk}} *(\omega)[12], \mathrm{T}_{\mathrm{i} \rightarrow \mathrm{f}}$ can be expressed (see Appendix A1) as

$$
\begin{aligned}
& \mathrm{T}_{\mathrm{i} \rightarrow \mathrm{f}}=\mathrm{T}_{\mathrm{i} \rightarrow \mathrm{f}}^{0}+\lim _{\varepsilon, \varepsilon^{\prime} \rightarrow 0}(2 \pi)^{-1}\left(\mathrm{t}_{\mathrm{f}}-\mathrm{t}_{\mathrm{i}}\right) \exp \left[-\mathrm{iE}_{0}\left(\mathrm{t}_{\mathrm{f}}-\mathrm{t}_{\mathrm{i}}\right) \hbar^{-1}\right] \int_{0}^{+\infty} \mathrm{d} \omega \sum_{\mathrm{ijkl}} \alpha_{\mathrm{ij}}(\omega)_{\mathrm{B}} \alpha_{\mathrm{kl}}(\omega)_{\mathrm{A}}
\end{aligned}
$$

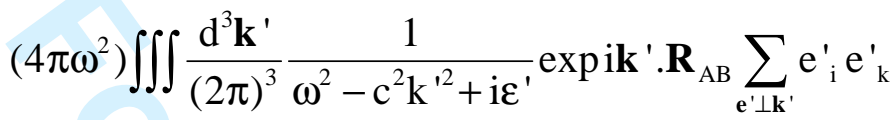

$$
\begin{aligned}
& \left(4 \pi \omega^{2}\right) \iiint \frac{d^{3} \mathbf{k}}{(2 \pi)^{3}} \frac{1}{\omega^{2}-c^{2} k^{2}+i \varepsilon} \exp -i \mathbf{k} \cdot \mathbf{R}_{\mathrm{AB}} \sum_{\mathbf{e} \perp \mathbf{k}} \mathrm{e}_{\mathrm{j}} \mathrm{e}_{1}
\end{aligned}
$$

That expression holds even if the polarisability is not purely real.

The integral on $\mathbf{k}$ can be calculated (see Appendix A1) as

$$
\begin{aligned}
& \lim _{\varepsilon \rightarrow 0} \iiint \frac{d^{3} \mathbf{k}}{(2 \pi)^{3}} \frac{1}{\omega^{2}-c^{2} k^{2}+i \varepsilon} \exp -i \mathbf{k} . \mathbf{R}_{A B} \sum_{\mathbf{e} \perp \mathbf{k}} e_{j} e_{1} \\
& =\delta_{j 1}\left(1-\left(\frac{i \omega R}{c}\right)+\left(\frac{i \omega R}{c}\right)^{2}\right)\left(\frac{\operatorname{expi\omega R/c}}{4 \pi \omega^{2} R^{3}}\right)-\frac{R_{j} R_{1}}{R^{2}}\left(3-3\left(\frac{i \omega R}{c}\right)+\left(\frac{i \omega R}{c}\right)^{2}\right)\left(\frac{\operatorname{expi\omega R} / c}{4 \pi \omega^{2} R^{3}}\right)
\end{aligned}
$$

Let us put

$$
\begin{aligned}
D_{j 1}(x) & =\delta_{j 1}\left(1-x+x^{2}\right) \exp (x)-\frac{R_{j} R_{1}}{R^{2}}\left(3-3 x+x^{2}\right) \exp (x) \\
& =\delta_{j 1} \frac{2}{3} x^{2} \exp (x)-\left(\frac{3 R_{j} R_{1}-R^{2} \delta_{j 1}}{2 R^{2}}\right) \frac{2}{3}\left(3-3 x+x^{2}\right) \exp (x)
\end{aligned}
$$

The resulting expression for the transition amplitude is

$$
\begin{aligned}
\mathrm{T}_{\mathrm{i} \rightarrow \mathrm{f}}=\mathrm{T}_{\mathrm{i} \rightarrow \mathrm{f}}^{0}+(2 \pi)^{-1}\left(\mathrm{t}_{\mathrm{f}}-\mathrm{t}_{\mathrm{i}}\right) \exp \left[-\mathrm{iE}_{0}\left(\mathrm{t}_{\mathrm{f}}-\mathrm{t}_{\mathrm{i}}\right) \hbar^{-1}\right] \\
\int_{0}^{+\infty} \mathrm{d} \omega \sum_{\mathrm{ijkl}} \alpha_{\mathrm{ij}}(\omega)_{\mathrm{B}} \alpha_{\mathrm{kl}}(\omega)_{\mathrm{A}} \mathrm{R}^{-6} \mathrm{D}_{\mathrm{ik}}(\mathrm{i} \omega \mathrm{R} / \mathrm{c}) \mathrm{D}_{\mathrm{jl}}(\mathrm{i} \omega \mathrm{R} / \mathrm{c})
\end{aligned}
$$

That expression can be written in a somehow more convenient way by putting $\omega=i \xi$, so that it becomes

$$
\begin{aligned}
\mathrm{T}_{\mathrm{i} \rightarrow \mathrm{f}}=\mathrm{T}_{\mathrm{i} \rightarrow \mathrm{f}}^{0}+\mathrm{i}(2 \pi)^{-1}\left(\mathrm{t}_{\mathrm{f}}-\mathrm{t}_{\mathrm{i}}\right) \exp \left[-\mathrm{iE}_{0}\left(\mathrm{t}_{\mathrm{f}}-\mathrm{t}_{\mathrm{i}}\right) \hbar^{-1}\right] \int_{0}^{+\infty} \mathrm{d} \xi \sum_{\mathrm{ijkl}} \alpha_{\mathrm{ij}}(\mathrm{i} \xi)_{\mathrm{B}} \alpha_{\mathrm{kl}}(\mathrm{i} \xi)_{\mathrm{A}} \\
\mathrm{R}^{-6} \mathrm{D}_{\mathrm{ik}}(-\xi \mathrm{R} / \mathrm{c}) \mathrm{D}_{\mathrm{jl}}(-\xi \mathrm{R} / \mathrm{c})
\end{aligned}
$$

From the explicit expression of $D_{j 1}(i \omega R / c)$, it appears that $D_{j 1}(-\xi R / c)$ is a real, non diverging function, and the same is true for $\alpha_{i j}(i \xi)$. 
Calling $\Delta \mathrm{E}$ the energy shift caused by the molecular interactions in such a way that

$$
\mathrm{T}_{\mathrm{i} \rightarrow \mathrm{f}}=\exp \left[-\mathrm{i}\left(\mathrm{E}_{0}+\Delta \mathrm{E}\right)\left(\mathrm{t}_{\mathrm{f}}-\mathrm{t}_{\mathrm{i}}\right) \hbar^{-1}\right] \cong\left[1-\mathrm{i} \Delta \mathrm{E}\left(\mathrm{t}_{\mathrm{f}}-\mathrm{t}_{\mathrm{i}}\right) \hbar^{-1}\right] \exp \left[-\mathrm{iE}_{0}\left(\mathrm{t}_{\mathrm{f}}-\mathrm{t}_{\mathrm{i}}\right) \hbar^{-1}\right]
$$

we obtain

$$
\Delta \mathrm{E}=-(2 \pi)^{-1} \int_{0}^{+\infty} \hbar \mathrm{d} \xi \sum_{\mathrm{ij}} \alpha_{\mathrm{ij}}(\mathrm{i} \xi)_{\mathrm{B}} \alpha_{\mathrm{kl}}(\mathrm{i} \xi)_{\mathrm{A}} \mathrm{R}^{-6} \mathrm{D}_{\mathrm{ik}}(-\xi \mathrm{R} / \mathrm{c}) \mathrm{D}_{\mathrm{j} 1}(-\xi \mathrm{R} / \mathrm{c})
$$

Now (see Appendix A2), $D_{j 1}$ as a function of $\mathbf{R}$ can be expressed as the sum of two fully symmetric (with respect to index permutations) tensors irreducible under the rotation group, a scalar one $(\mathrm{J}=0)$ and a quadrupolar one $(\mathrm{J}=2)$. Consequently $\mathrm{D}_{\mathrm{ik}} \mathrm{D}_{\mathrm{jl}}$ is the sum of a scalar term, a quadrupolar term, and a hexadecapolar term $(\mathrm{J}=4)$, among which, assuming an isotropic distribution of the molecular centres, we will only retain the scalar contribution $\left\{\mathrm{D}_{\mathrm{ik}} \mathrm{D}_{\mathrm{j} 1}\right\}^{(0)}$.

Introducing the two orthonormal, fully symmetric, Cartesian tensors (see Appendix A2)

$$
\mathfrak{S}_{\mathrm{j} 1}=\frac{\delta_{\mathrm{j} 1}}{\sqrt{3}}
$$

and

$$
\mathfrak{D}_{\mathrm{j} 1}=\frac{3 \mathrm{R}_{\mathrm{j}} \mathrm{R}_{1}-\mathrm{R}^{2} \delta_{\mathrm{j} 1}}{\sqrt{6} \mathrm{R}^{2}}
$$

we can write

$$
D_{j 1}(x)=\mathfrak{S}_{j 1} \frac{1}{\sqrt{3}} 2 x^{2} \exp (x)-\mathfrak{D}_{j 1} \sqrt{\frac{2}{3}}\left(3-3 x+x^{2}\right) \exp (x)
$$

and hence

$$
\begin{gathered}
\left\{\mathrm{D}_{\mathrm{ik}} \mathrm{D}_{\mathrm{j} 1}\right\}^{(0)}=\left(\frac{2 \mathrm{x}^{2} \exp (\mathrm{x})}{\sqrt{3}}\right)^{2} \mathfrak{S}_{\mathrm{ik}} \mathfrak{S}_{\mathrm{j} 1}+\left(\frac{\sqrt{2}\left(3-3 \mathrm{x}+\mathrm{x}^{2}\right) \exp (\mathrm{x})}{\sqrt{3}}\right)^{2} \mathfrak{S}_{\mathrm{ik}} \mathfrak{S}_{\mathrm{j} 1} \\
=\delta_{\mathrm{ik}} \delta_{\mathrm{j} 1} \frac{2}{3}\left(3-6 \mathrm{x}+5 \mathrm{x}^{2}-2 \mathrm{x}^{3}+\mathrm{x}^{4}\right) \exp (2 \mathrm{x})
\end{gathered}
$$

Finally one gets

$$
\begin{aligned}
\Delta \mathrm{E}=- & \int_{0}^{+\infty} \hbar \mathrm{d} \xi \sum_{\mathrm{ij}} \alpha_{\mathrm{ij}}(\mathrm{i} \xi)_{\mathrm{B}} \alpha_{\mathrm{ij}}(\mathrm{i} \xi)_{\mathrm{A}} \\
& \frac{1}{\pi \mathrm{R}^{6}}\left(1+\frac{2 \xi \mathrm{R}}{\mathrm{c}}+\frac{5 \xi^{2} \mathrm{R}^{2}}{2 \mathrm{c}^{2}}+\frac{\xi^{3} \mathrm{R}^{3}}{\mathrm{c}^{3}}+\frac{\xi^{4} \mathrm{R}^{4}}{2 \mathrm{c}^{4}}\right) \exp (-2 \xi \mathrm{R} / \mathrm{c})
\end{aligned}
$$

$\sum_{\mathrm{ij}} \alpha_{\mathrm{ij}}(\mathrm{i} \xi)_{\mathrm{B}} \alpha_{\mathrm{ij}}(\mathrm{i} \xi)_{\mathrm{A}}=\left(\alpha(\mathrm{i} \xi)_{\mathrm{B}} \cdot \alpha(\mathrm{i} \xi)_{\mathrm{A}}\right)$ is the scalar product of the two polarisability tensors $\alpha_{\mathrm{A}}$ and $\alpha_{\mathrm{B}} \cdot \alpha_{\mathrm{ij}}$ is a rank 2 true tensor, it has a priori three irreducible parts, a symmetric (with respect to index permutations) true scalar, a symmetric true quadrupole and an anti-symmetric 


\section{Beyond the electric dipole (E1) approximation}

\section{One M1 or E2 vortex}

In the previous section, we had considered that the four interaction vortices were of electric dipole type (E1). In the present part, we will go beyond that approximation. The next order term beyond the electric dipole coupling is the electric quadrupole (E2) + magnetic dipole (M1) couplings. If among the 4 interaction vortices one is of M1 type, we then have to change on that vortex d.e by $\omega^{-1} \mathbf{M} . \mathbf{k} \times \mathbf{e}$, i.e. $\mathrm{d}_{1} \mathrm{e}_{1}$ by $\varepsilon_{\mathrm{pq}} \omega^{-1} \mathrm{M}_{\mathrm{p}} \mathrm{k}_{\mathrm{q}} \mathrm{e}_{1}$. Alternatively, if among the 4 interaction vortices one is of $\mathrm{E} 2$ type, we have to change on that vortex d.e by either $\frac{\mathrm{i}}{2} \mathbf{Q}: \mathbf{k e}$ (photon absorption) or by $\frac{-\mathrm{i}}{2} \mathbf{Q}: \mathbf{k e}$ (photon emission), i.e. to replace $\mathrm{d}_{1} \mathrm{e}_{1}$ by $\frac{ \pm \mathrm{i}}{2} \mathrm{Q}_{\mathrm{q}} \mathrm{k}_{\mathrm{q}} \mathrm{e}_{1}$. An extra term $\Lambda_{\mathrm{i} \rightarrow \mathrm{f}}$ has thus to be added in the expression of the transition amplitude $\mathrm{T}_{\mathrm{i} \rightarrow \mathrm{f}}$ equ.(2), which is found to be equal to 


$$
\begin{aligned}
& \Lambda_{\mathrm{i} \rightarrow \mathrm{f}}=\lim _{\varepsilon, \varepsilon^{\prime} \rightarrow 0}(2 \pi)^{-1}\left(\mathrm{t}_{\mathrm{f}}-\mathrm{t}_{\mathrm{i}}\right) \exp \left[-\mathrm{iE}_{0}\left(\mathrm{t}_{\mathrm{f}}-\mathrm{t}_{\mathrm{i}}\right) \hbar^{-1}\right] \int_{0}^{+\infty} \omega^{4} \mathrm{~d} \omega(4 \pi)^{2} \sum_{\mathrm{ijklq}} \alpha_{\mathrm{ij}}(\omega)_{\mathrm{B}} \mathrm{i}^{-1} \eta_{\mathrm{kql}}(\omega)_{\mathrm{A}} \\
& \iiint \frac{d^{3} \mathbf{k}^{\prime}}{(2 \pi)^{3}} \frac{1}{\omega^{2}-c^{2} k^{\prime 2}+i \varepsilon^{\prime}} \operatorname{expik} \mathbf{k}^{\prime} \cdot \mathbf{R}_{\mathrm{AB}} \\
& \iiint \frac{d^{3} \mathbf{k}}{(2 \pi)^{3}} \frac{1}{\omega^{2}-c^{2} k^{2}+i \varepsilon} \exp -i \mathbf{k} \cdot \mathbf{R}_{\mathrm{AB}} \\
& \sum_{\mathbf{e}^{\prime} \perp \mathbf{k}^{\prime}} \mathrm{e}_{\mathrm{i}}^{{ }^{\prime}} \mathrm{e}^{\prime}{ }_{\mathrm{k}} \sum_{\mathbf{e} \perp \mathbf{k}} \mathrm{e}_{\mathrm{j}} \mathrm{k}_{\mathrm{q}} \mathrm{e}_{1} \\
& + \text { a similar term with the exchange of } \mathrm{A} \text { and } \mathrm{B}
\end{aligned}
$$

where

$$
\begin{aligned}
\eta_{\text {ipj }}(\omega)= & \left(4 \pi \epsilon_{0}\right)^{-1} \sum_{n} \frac{\left\langle 0\left|d_{i} P_{n}\left[i Q_{p j}+\omega^{-1} \varepsilon_{q p j} M_{q}\right]\right| 0\right\rangle}{E_{0}-E_{n}+\hbar \omega-\Delta_{n}(\omega)+\frac{i \Gamma_{n}(\omega)}{2}}-\frac{\left\langle 0\left|\left[i Q_{i p}-i \omega^{-1} \varepsilon_{q i p} M_{q}\right] P_{n} d_{j}\right| 0\right\rangle}{E_{0}-E_{n}+\hbar \omega-\Delta_{n}(\omega)+\frac{i \Gamma_{n}(\omega)}{2}} \\
& +\frac{\left\langle 0\left|\left[i Q_{j p}+\omega^{-1} \varepsilon_{q j} M_{q}\right] P_{n} d_{i}\right| 0\right\rangle}{E_{0}-E_{n}-\hbar \omega-\Delta_{n}(-\omega)-\frac{i \Gamma_{n}(-\omega)}{2}}-\frac{\left\langle 0\left|d_{j} P_{n}\left[i Q_{p i}-\omega^{-1} \varepsilon_{q p i} M_{q}\right]\right| 0\right\rangle}{E_{0}-E_{n}-\hbar \omega-\Delta_{n}(-\omega)-\frac{i \Gamma_{n}(-\omega)}{2}}
\end{aligned}
$$

is the molecular optical activity tensor, which exists only in chiral molecules; so the fact that it may be involved in the interactions between chiral molecules is not surprising.

We will have to compute as previously

$$
\lim _{\varepsilon \rightarrow 0} \iiint \frac{d^{3} \mathbf{k}}{(2 \pi)^{3}} \frac{1}{\omega^{2}-c^{2} k^{2}+i \varepsilon} \exp -i \mathbf{k} . \mathbf{R}_{\mathrm{AB}} \sum_{\mathbf{e} \perp \mathbf{k}} \mathrm{e}_{\mathrm{j}} \mathrm{e}_{1}=\frac{1}{4 \pi \omega^{2} \mathrm{R}^{3}} \mathrm{D}_{\mathrm{jl}}(\mathrm{i} \omega \mathrm{R} / \mathrm{c})
$$

but also

$$
\lim _{\varepsilon \rightarrow 0} \iiint \frac{d^{3} \mathbf{k}}{(2 \pi)^{3}} \frac{1}{\omega^{2}-c^{2} k^{2}+i \varepsilon} \exp -i \mathbf{k} \cdot \mathbf{R}_{A B} \sum_{\mathbf{e} \perp \mathbf{k}} \mathrm{e}_{j} k_{q} e_{1}
$$

which is readily seen to be

$$
\begin{gathered}
\mathrm{i} \nabla_{\mathrm{q}} \lim _{\varepsilon \rightarrow 0} \iiint \frac{\mathrm{d}^{3} \mathbf{k}}{(2 \pi)^{3}} \frac{1}{\omega^{2}-\mathrm{c}^{2} \mathrm{k}^{2}+\mathrm{i} \varepsilon} \exp -\mathrm{ik} \cdot \mathbf{R}_{\mathrm{AB}} \sum_{\mathbf{e} \perp \mathbf{k}} \mathrm{e}_{\mathrm{j}} \mathrm{e}_{1} \\
\quad=\mathrm{i} \nabla_{\mathrm{q}} \frac{1}{4 \pi \omega^{2} \mathrm{R}^{3}} \mathrm{D}_{\mathrm{j} 1}(\mathrm{i} \omega \mathrm{R} / \mathrm{c})
\end{gathered}
$$

Let us put

$$
\mathrm{R}^{-4} \mathrm{~F}_{\mathrm{jlq}}(\mathrm{i} \omega \mathrm{R} / \mathrm{c})=\nabla_{\mathrm{q}} \frac{1}{\mathrm{R}^{3}} \mathrm{D}_{\mathrm{jl}}(\mathrm{i} \omega \mathrm{R} / \mathrm{c})
$$


The rank 3 cartesian tensor $\mathrm{F}_{\mathrm{jlq}}$ can be expressed as the sum of irreducible tensors of respective orders $\mathrm{J}=1$ and $\mathrm{J}=3$ due to the odd number of $\mathrm{k}$ or $\nabla$ vector components. Combining equ.(17) and equ.(19) a priori produces $\mathrm{J}=1, \mathrm{~J}=3$ and $\mathrm{J}=5$ terms, but no scalar $(J=0)$. Actually, the rank-3 true tensor $\eta_{\text {ipj }}(\omega)$ is anti-symmetric in the exchange of its extreme indices $\mathrm{i}$ and $\mathrm{j}$ from a general property of the kinetic coefficients and thus it is equivalent to a rank-2 pseudo-tensor $\gamma$. As a consequence, $\mathrm{J}$ cannot be higher than 4 so that only the $\mathrm{J}=1$ and $\mathrm{J}=3$ terms are effective. If the molecules are randomly distributed, the absence of a $\mathrm{J}=0$ term causes the above expression equ.(15) not to contribute to the molecular interaction energy.

\section{Two M1 or E2 vortices}

If we have two interaction vortices of M1 or E2 type, there can be an isotropic term because of the even number of k-vector occurrences.

We have then to consider 3 types of diagrams.

(1) There is one M1 or E2 vortex on each molecule, but they refer to a different photon:

$$
\begin{aligned}
& \Lambda_{\mathrm{i} \rightarrow \mathrm{f}}=\lim _{\varepsilon, \varepsilon^{\prime} \rightarrow 0}-(2 \pi)^{-1}\left(\mathrm{t}_{\mathrm{f}}-\mathrm{t}_{\mathrm{i}}\right) \exp \left[-\mathrm{iE}_{0}\left(\mathrm{t}_{\mathrm{f}}-\mathrm{t}_{\mathrm{i}}\right) \hbar^{-1}\right] \int_{0}^{+\infty} \mathrm{d} \omega \sum_{\mathrm{j} j \mathrm{klpq}} \eta_{\mathrm{ijp}}(\omega)_{\mathrm{B}} \eta_{\mathrm{klq}}(\omega)_{\mathrm{A}} \\
& \left(4 \pi \omega^{2}\right) \iiint \frac{\mathrm{d}^{3} \mathbf{k}^{\prime}}{(2 \pi)^{3}} \frac{1}{\omega^{2}-\mathrm{c}^{2} \mathrm{k}^{\prime 2}+\mathrm{i} \varepsilon^{\prime}} \exp \mathbf{k}^{\prime} \cdot \mathbf{R}_{\mathrm{AB}} \sum_{\mathrm{e}^{\prime} \perp \mathbf{k}^{\prime}} \mathrm{e}_{\mathrm{i}}{ }_{\mathrm{i}} \mathrm{k}_{\mathrm{p}}{ }_{\mathrm{p}} \mathrm{e}^{\prime}{ }_{\mathrm{k}} \\
& \left(4 \pi \omega^{2}\right) \iiint \frac{d^{3} \mathbf{k}}{(2 \pi)^{3}} \frac{1}{\omega^{2}-c^{2} k^{2}+i \varepsilon} \exp -i \mathbf{k} \cdot \mathbf{R}_{A B} \sum_{\mathbf{e} \perp \mathbf{k}} \mathrm{e}_{\mathrm{j}} \mathrm{k}_{\mathrm{q}} \mathrm{e}_{1}
\end{aligned}
$$

Using equs.(3) and (4), that expression becomes

$$
\begin{aligned}
& \Lambda_{\mathrm{i} \rightarrow \mathrm{f}}=-(2 \pi)^{-1}\left(\mathrm{t}_{\mathrm{f}}-\mathrm{t}_{\mathrm{i}}\right) \exp \left[-\mathrm{iE}_{0}\left(\mathrm{t}_{\mathrm{f}}-\mathrm{t}_{\mathrm{i}}\right) \hbar^{-1}\right] \int_{0}^{+\infty} \mathrm{d} \omega \sum_{\mathrm{ijk} k \mathrm{pq}} \eta_{\mathrm{ijp}}(\omega)_{\mathrm{B}} \eta_{\mathrm{klq}}(\omega)_{\mathrm{A}} \\
&\left(-\mathrm{i} \nabla_{\mathrm{p}} \frac{1}{\mathrm{R}^{3}} \mathrm{D}_{\mathrm{ik}}(\mathrm{i} \omega \mathrm{R} / \mathrm{c})\right)\left(\mathrm{i} \nabla_{\mathrm{q}} \frac{1}{\mathrm{R}^{3}} \mathrm{D}_{\mathrm{j} \mathrm{l}}(\mathrm{i} \omega \mathrm{R} / \mathrm{c})\right) \\
&=-(2 \pi)^{-1}\left(\mathrm{t}_{\mathrm{f}}-\mathrm{t}_{\mathrm{i}}\right) \exp \left[-\mathrm{iE}_{0}\left(\mathrm{t}_{\mathrm{f}}-\mathrm{t}_{\mathrm{i}}\right) \hbar^{-1}\right] \int_{0}^{+\infty} \mathrm{d} \omega \sum_{\mathrm{ijklpq}} \eta_{\mathrm{ijp}}(\omega)_{\mathrm{B}} \eta_{\mathrm{klq}}(\omega)_{\mathrm{A}} \\
&\left(\frac{1}{\mathrm{R}^{4}} \mathrm{~F}_{\mathrm{ikp}}(\mathrm{i} \omega \mathrm{R} / \mathrm{c})\right)\left(\frac{1}{\mathrm{R}^{4}} \mathrm{~F}_{\mathrm{jlq}}(\mathrm{i} \omega \mathrm{R} / \mathrm{c})\right)
\end{aligned}
$$

The combination of $\mathrm{F}_{\mathrm{ikp}}$ and $\mathrm{F}_{\mathrm{jlq}}$ produces a scalar contribution, as well as contributions of orders $\mathrm{J}=2, \mathrm{~J}=4$, and $\mathrm{J}=6$. Equ. (21) leads to an interaction energy of the following form:

$$
\Delta \mathrm{E}(1)=+(2 \pi)^{-1} \int_{0}^{+\infty} \hbar \mathrm{d} \xi \sum_{\mathrm{ijklpq}} \eta_{\mathrm{ijp}}(\mathrm{i} \xi)_{\mathrm{B}} \eta_{\mathrm{klq}}(\mathrm{i} \xi)_{\mathrm{A}} \mathrm{R}^{-8} \mathrm{~F}_{\mathrm{ikp}}(-\xi \mathrm{R} / \mathrm{c}) \mathrm{F}_{\mathrm{jlq}}(-\xi \mathrm{R} / \mathrm{c})
$$

In the short distance limit where the retardation effects can be neglected ( $\xi \mathrm{R} / \mathrm{c}<<1)$, we had noticed earlier that $D_{j 1}$ is reduced to a pure quadrupole. That implies that $F_{j l q}$ is then reduced to a pure octopole, fully symmetric with respect to index permutations (see Appendix A2) since 


$$
\mathrm{R}^{-4} \mathrm{~F}_{\mathrm{jlq}} \rightarrow \nabla_{\mathrm{q}} \frac{1}{\mathrm{R}^{3}}\left(\frac{-3 \mathrm{R}_{\mathrm{j}} \mathrm{R}_{1}+\mathrm{R}^{2} \delta_{\mathrm{jl}}}{\mathrm{R}^{2}}\right)=\frac{3}{\mathrm{R}^{4}}\left(\frac{5 \mathrm{R}_{\mathrm{j}} \mathrm{R}_{1} \mathrm{R}_{\mathrm{q}}-\mathrm{R}_{\mathrm{j}} \mathrm{R}^{2} \delta_{\mathrm{lq}}-\mathrm{R}_{1} \mathrm{R}^{2} \delta_{\mathrm{jq}}-\mathrm{R}_{\mathrm{q}} \mathrm{R}^{2} \delta_{\mathrm{lj}}}{\mathrm{R}^{3}}\right)
$$

Introducing the fully symmetric tensor with a unity norm (see Appendix A2)

$$
\mathfrak{F}_{\mathrm{jlq}}=\frac{5 \mathrm{R}_{\mathrm{j}} \mathrm{R}_{\mathrm{l}} \mathrm{R}_{\mathrm{q}}-\mathrm{R}_{\mathrm{j}} \mathrm{R}^{2} \delta_{\mathrm{lq}}-\mathrm{R}_{\mathrm{l}} \mathrm{R}^{2} \delta_{\mathrm{jq}}-\mathrm{R}_{\mathrm{q}} \mathrm{R}^{2} \delta_{\mathrm{lj}}}{2 \mathrm{R}^{3}}
$$

we get

$$
\mathrm{F}_{\mathrm{jlq}} \rightarrow \frac{6}{\mathrm{R}^{4}} \mathfrak{F}_{\mathrm{jlq}}
$$

With randomly distributed molecules, we only retain the scalar part of $\mathrm{F}_{\mathrm{ikp}} \mathrm{F}_{\mathrm{jlq}}$, i.e.

$$
\left\{\mathrm{F}_{\mathrm{ikp}} \mathrm{F}_{\mathrm{jlq}}\right\}^{(0)} \rightarrow \frac{36}{\mathrm{R}^{8}}\|\mathfrak{F}\|^{2} \delta_{\mathrm{ij}} \delta_{\mathrm{kl}} \delta_{\mathrm{pq}}=\frac{36}{\mathrm{R}^{8}} \delta_{\mathrm{ij}} \delta_{\mathrm{kl}} \delta_{\mathrm{pq}}
$$

We had pointed out earlier that $\eta_{\text {ipj }}$ being anti-symmetric in the permutation of its extreme indices $\mathrm{i}$ and $\mathrm{j}$ is equivalent to a rank 2 pseudo-tensor $\gamma \cdot \gamma$ is obtained from $\eta_{\mathrm{ipj}}=\sum_{\mathrm{q}} \gamma_{\mathrm{pq}} \varepsilon_{\mathrm{iqj}}$, i.e. $\gamma_{\mathrm{xy}}=\eta_{\mathrm{xxz}}, \gamma_{\mathrm{yx}}=-\eta_{\mathrm{yyz}}, \gamma_{\mathrm{zz}}=\eta_{\mathrm{yzx}}$. $\eta_{\text {ipj }}$ has a fully antisymmetric (with respect to index permutations) pseudo-scalar part $\left\{1^{3}\right\} \eta^{(0)}$, one true vector part $\{21\} \eta^{(1)}$ and one pseudo-quadrupolar part $\{21\} \eta^{(2)}$ (see Appendix A2). The pseudo-scalar part of $\eta$ is the trace of $\gamma$ :

$$
\eta_{\mathrm{ijp}}^{(0)}=\bar{\eta} \varepsilon_{\mathrm{ijp}} \text { with } \bar{\eta}=\frac{1}{3} \sum_{\mathrm{pq}} \delta_{\mathrm{pq}} \gamma_{\mathrm{pq}}=\frac{1}{3} \sum_{\mathrm{pqr}} \varepsilon_{\mathrm{pqr}} \eta_{\mathrm{pqr}}=\frac{1}{3}\left(\eta_{\mathrm{xyz}}+\eta_{\mathrm{yzx}}+\eta_{\mathrm{zxy}}\right)
$$

As the scalar part $\eta_{\text {ijp }}^{(0)}$ of $\eta_{\mathrm{ijp}}$ simply is $\bar{\eta} \varepsilon_{\mathrm{ijp}}$, the contribution of $\eta^{(0)}{ }_{\mathrm{ijp}}$ to $\Delta \mathrm{E}(1)$ involves the term $(\bar{\eta})^{2} \varepsilon_{\mathrm{ijp}} \varepsilon_{\mathrm{klq}} \mathrm{F}_{\mathrm{ikp}} \mathrm{F}_{\mathrm{jlq}}$. F being in the short distance limit fully symmetric with respect to index permutations, that term vanishes. It means that the pseudo-scalar part of the optical activity does not contribute significantly to $\Delta \mathrm{E}(1)$ (N.B.: that pseudo-scalar is entirely due to the magnetic term since $\left.\eta^{(0)}{ }_{\mathrm{ijp}} \nabla_{\mathrm{j}} \mathrm{E}_{\mathrm{p}}=\bar{\eta} \varepsilon_{\mathrm{ijp}} \nabla_{\mathrm{j}} \mathrm{E}_{\mathrm{p}}=\bar{\eta}(\boldsymbol{\nabla} \times \mathbf{E})_{\mathrm{i}}=\bar{\eta}\left(-\partial \mathbf{B}_{\mathrm{i}} / \partial \mathrm{t}\right)\right)$. Moreover, because of the transverse character of the electric field $\left(\nabla \cdot \mathbf{E}_{\perp}=0\right)$, the vector part $\eta^{(1)}{ }_{\mathrm{ijp}}$ of $\eta_{\mathrm{ijp}}$, whose $\mathrm{i}^{\text {th }}$ component is proportional to $\sum_{\mathrm{p}} \eta_{\mathrm{ipp}}$, vanishes.

We finally get

$$
\Delta \mathrm{E}(1) \rightarrow+\int_{0}^{+\infty} \frac{18 \hbar}{\pi \mathrm{R}^{8}} \mathrm{~d} \xi\left(\eta^{(2)}(\mathrm{i} \xi)_{\mathrm{B}} \cdot \eta^{(2)}(\mathrm{i} \xi)_{\mathrm{A}}\right)
$$

(2) There is one M1 or E2 vortex on each molecule, both vortices referring to the same photon: 


$$
\begin{gathered}
\Lambda_{\mathrm{i} \rightarrow \mathrm{f}}=-\lim _{\varepsilon, \varepsilon^{\prime} \rightarrow 0}(2 \pi)^{-1}\left(\mathrm{t}_{\mathrm{f}}-\mathrm{t}_{\mathrm{i}}\right) \exp \left[-\mathrm{iE}_{0}\left(\mathrm{t}_{\mathrm{f}}-\mathrm{t}_{\mathrm{i}}\right) \hbar^{-1}\right] \int_{0}^{+\infty} \omega^{4} \mathrm{~d} \omega \sum_{\mathrm{ijklpq}} \eta_{\mathrm{ijp}}(\omega)_{\mathrm{B}} \eta_{\mathrm{klq}}(\omega)_{\mathrm{A}} \\
(4 \pi) \iiint \frac{\mathrm{d}^{3} \mathbf{k}^{\prime}}{(2 \pi)^{3}} \frac{1}{\omega^{2}-\mathrm{c}^{2} \mathrm{k}^{\prime 2}+\mathrm{i} \varepsilon} \exp ^{\prime} \mathbf{k}^{\prime} \cdot \mathbf{R}_{\mathrm{AB}} \sum_{\mathrm{ijk} \mathrm{kl}} \sum_{\mathbf{e}^{\prime} \perp \mathbf{k}^{\prime}} \mathrm{e}_{\mathrm{i}}^{\prime} \mathrm{e}_{\mathrm{k}}^{\prime} \\
(4 \pi) \iiint \frac{\mathrm{d}^{3} \mathbf{k}}{(2 \pi)^{3}} \frac{1}{\omega^{2}-\mathrm{c}^{2} \mathrm{k}^{2}+\mathrm{i} \varepsilon} \exp -\mathrm{ik} \cdot \mathbf{R}_{\mathrm{AB}} \sum_{\mathbf{e} \perp \mathbf{k}} \mathrm{e}_{\mathrm{j}} \mathrm{e}_{1} \mathrm{k}_{\mathrm{p}} \mathrm{k}_{\mathrm{q}}
\end{gathered}
$$

That equation becomes

$$
\begin{aligned}
\Lambda_{\mathrm{i} \rightarrow \mathrm{f}}= & -(2 \pi)^{-1}\left(\mathrm{t}_{\mathrm{f}}-\mathrm{t}_{\mathrm{i}}\right) \exp \left[-\mathrm{iE}_{0}\left(\mathrm{t}_{\mathrm{f}}-\mathrm{t}_{\mathrm{i}}\right) \hbar^{-1}\right] \int_{0}^{+\infty} \mathrm{d} \omega \sum_{\mathrm{ijklpq}} \eta_{\mathrm{ijp}}(\omega)_{\mathrm{B}} \eta_{\mathrm{klq}}(\omega)_{\mathrm{A}} \\
& \left(\frac{1}{\mathrm{R}^{3}} \mathrm{D}_{\mathrm{ik}}(\mathrm{i} \omega \mathrm{R} / \mathrm{c})\right)\left(-\nabla_{\mathrm{p}} \nabla_{\mathrm{q}} \frac{1}{\mathrm{R}^{3}} \mathrm{D}_{\mathrm{jl}}(\mathrm{i} \omega \mathrm{R} / \mathrm{c})\right) \\
= & -(2 \pi)^{-1}\left(\mathrm{t}_{\mathrm{f}}-\mathrm{t}_{\mathrm{i}}\right) \exp \left[-\mathrm{iE}_{0}\left(\mathrm{t}_{\mathrm{f}}-\mathrm{t}_{\mathrm{i}}\right) \hbar^{-1}\right] \int_{0}^{+\infty} \mathrm{d} \omega \sum_{\mathrm{ijklpq}} \eta_{\mathrm{ijp}}(\omega)_{\mathrm{B}} \eta_{\mathrm{klq}}(\omega)_{\mathrm{A}} \\
& \left(\frac{1}{\mathrm{R}^{3}} \mathrm{D}_{\mathrm{ik}}(\mathrm{i} \omega \mathrm{R} / \mathrm{c})\right)\left(\frac{1}{\mathrm{R}^{5}} \mathrm{G}_{\mathrm{jlpq}}(\mathrm{i} \omega \mathrm{R} / \mathrm{c})\right)
\end{aligned}
$$

with

$\mathrm{R}^{-5} \mathrm{G}_{\mathrm{jlpq}}(\mathrm{i} \omega \mathrm{R} / \mathrm{c})=-\nabla_{\mathrm{p}} \nabla_{\mathrm{q}} \frac{1}{\mathrm{R}^{3}} \mathrm{D}_{\mathrm{jl}}(\mathrm{i} \omega \mathrm{R} / \mathrm{c})$

$\mathrm{G}_{\mathrm{jlpq}}$ is a rank 4 tensor which can be expressed as the sum of irreducible tensors of orders $\mathrm{J}=0, \mathrm{~J}=2$ and $\mathrm{J}=4$. Hence the combination of $\mathrm{D}_{\mathrm{ik}}$ and $\mathrm{G}_{\mathrm{jlpq}}$ produces contributions of order $\mathrm{J}=0, \mathrm{~J}=2, \mathrm{~J}=4$ and $\mathrm{J}=6$. Equ.(27) leads to an interaction energy of the following form:

$$
\Delta \mathrm{E}(2)=(2 \pi)^{-1} \int_{0}^{+\infty} \hbar \mathrm{d} \xi \sum_{\mathrm{ijklpq}} \eta_{\mathrm{ijp}}(i \xi)_{\mathrm{B}} \eta_{\mathrm{klq}}(\mathrm{i} \xi)_{\mathrm{A}} \mathrm{R}^{-8} \mathrm{D}_{\mathrm{ik}}(-\xi \mathrm{R} / \mathrm{c}) \mathrm{G}_{\mathrm{jlpq}}(-\xi \mathrm{R} / \mathrm{c}) .
$$

In the short distance limit $\mathrm{G}_{\mathrm{jlpq}}$ is reduced to a pure hexadecapole ( $\left.\mathrm{J}=4\right)$, fully symmetric with respect to index permutations (see Appendix A2), since

$$
\begin{aligned}
\mathrm{G}_{\mathrm{jlpq}} \rightarrow & \nabla_{\mathrm{p}} \nabla_{\mathrm{q}} \frac{1}{\mathrm{R}^{3}}\left(\frac{3 \mathrm{R}_{\mathrm{j}} \mathrm{R}_{1}-\mathrm{R}^{2} \delta_{\mathrm{jl}}}{\mathrm{R}^{2}}\right) \\
& =\frac{3}{\mathrm{R}^{5}} \frac{\left(\begin{array}{r}
35 \mathrm{R}_{\mathrm{j}} \mathrm{R}_{\mathrm{l}} \mathrm{R}_{\mathrm{p}} \mathrm{R}_{\mathrm{q}}-5 \mathrm{R}_{\mathrm{j}} \mathrm{R}_{\mathrm{q}} \mathrm{R}^{2} \delta_{\mathrm{pl}}-5 \mathrm{R}_{\mathrm{j}} \mathrm{R}_{\mathrm{p}} \mathrm{R}^{2} \delta_{\mathrm{q} 1}-5 \mathrm{R}_{\mathrm{j}} \mathrm{R}_{1} \mathrm{R}^{2} \delta_{\mathrm{pq}}-5 \mathrm{R}_{\mathrm{p}} \mathrm{R}_{\mathrm{q}} \mathrm{R}^{2} \delta_{\mathrm{jl}} \\
-5 \mathrm{R}_{\mathrm{p}} \mathrm{R}_{\mathrm{l}} \mathrm{R}^{2} \delta_{\mathrm{jq}}-5 \mathrm{R}_{\mathrm{q}} \mathrm{R}_{\mathrm{l}} \mathrm{R}^{2} \delta_{\mathrm{jp}}+\mathrm{R}^{4} \delta_{\mathrm{jl}} \delta_{\mathrm{pq}}+\mathrm{R}^{4} \delta_{\mathrm{jp}} \delta_{\mathrm{lq}}+\mathrm{R}^{4} \delta_{\mathrm{jq}} \delta_{\mathrm{lp}}
\end{array}\right)}{\mathrm{R}^{4}}
\end{aligned}
$$

Introducing the fully symmetric tensor with a unity norm (see Appendix A2)

$$
\mathfrak{G}_{\mathrm{jlpq}}=\frac{\begin{array}{c}
35 \mathrm{R}_{\mathrm{j}} \mathrm{R}_{\mathrm{l}} \mathrm{R}_{\mathrm{p}} \mathrm{R}_{\mathrm{q}}-5 \mathrm{R}_{\mathrm{j}} \mathrm{R}_{\mathrm{q}} \mathrm{R}^{2} \delta_{\mathrm{pl}}-5 \mathrm{R}_{\mathrm{j}} \mathrm{R}_{\mathrm{p}} \mathrm{R}^{2} \delta_{\mathrm{q} 1}-5 \mathrm{R}_{\mathrm{j}} \mathrm{R}_{\mathrm{l}} \mathrm{R}^{2} \delta_{\mathrm{pq}}-5 \mathrm{R}_{\mathrm{p}} \mathrm{R}_{\mathrm{q}} \mathrm{R}^{2} \delta_{\mathrm{jl}} \\
-5 \mathrm{R}_{\mathrm{p}} \mathrm{R}_{\mathrm{l}} \mathrm{R}^{2} \delta_{\mathrm{jq}}-5 \mathrm{R}_{\mathrm{q}} \mathrm{R}_{\mathrm{l}} \mathrm{R}^{2} \delta_{\mathrm{jp}}+\mathrm{R}^{4} \delta_{\mathrm{jl}} \delta_{\mathrm{pq}}+\mathrm{R}^{4} \delta_{\mathrm{jp}} \delta_{\mathrm{lq}}+\mathrm{R}^{4} \delta_{\mathrm{jq}} \delta_{\mathrm{lp}}
\end{array}}{8 \mathrm{R}^{4}}
$$


we get $\mathrm{G}_{\mathrm{jlpq}} \rightarrow \frac{24}{\mathrm{R}^{5}} \mathfrak{G}_{\mathrm{jlpq}}$.

Consequently with randomly distributed molecules the coupling of $\mathrm{G}_{\mathrm{jlpq}}$ and $\mathrm{D}_{\mathrm{ik}}$ cannot produce a scalar if the centres of the molecules are uniformly distributed, hence the contribution of $\Delta \mathrm{E}(2)$ is presumably very weak with respect to $\Delta \mathrm{E}(1)$.

(3) Both M1 or E2 vortices are on the same molecule:

$$
\begin{gathered}
\Lambda_{\mathrm{i} \rightarrow \mathrm{f}}=\lim _{\varepsilon, \varepsilon^{\prime} \rightarrow 0}(2 \pi)^{-1}\left(\mathrm{t}_{\mathrm{f}}-\mathrm{t}_{\mathrm{i}}\right) \exp \left[-\mathrm{iE}_{0}\left(\mathrm{t}_{\mathrm{f}}-\mathrm{t}_{\mathrm{i}}\right) \hbar^{-1}\right] \int_{0}^{+\infty} \mathrm{d} \omega \sum_{\mathrm{ijklpq}} \sigma_{\mathrm{ijpq}}(\omega)_{\mathrm{B}} \alpha_{\mathrm{kl}}(\omega)_{\mathrm{A}} \\
\left(4 \pi \omega^{2}\right) \iiint \frac{\mathrm{d}^{3} \mathbf{k}^{\prime}}{(2 \pi)^{3}} \frac{1}{\omega^{2}-\mathrm{c}^{2} \mathrm{k}^{\prime 2}+\mathrm{i} \varepsilon^{\prime}} \operatorname{expik}^{\prime} \cdot \mathbf{R}_{\mathrm{AB}} \sum_{\mathrm{e}^{\prime} \perp \mathbf{k}^{\prime}} \mathrm{e}_{\mathrm{i}}^{\prime} \mathrm{k}_{\mathrm{p}}^{\prime} \mathrm{e}_{\mathrm{k}}^{\prime} \\
\left(4 \pi \omega^{2}\right) \iiint \frac{\mathrm{d}^{3} \mathbf{k}}{(2 \pi)^{3}} \frac{1}{\omega^{2}-\mathrm{c}^{2} \mathrm{k}^{2}+\mathrm{i} \varepsilon} \exp -\mathrm{ik} \cdot \mathbf{R}_{\mathrm{AB}} \sum_{\mathbf{e} \perp \mathbf{k}} \mathrm{e}_{\mathrm{j}} \mathrm{k}_{\mathrm{q}} \mathrm{e}_{1} \\
+ \text { a similar term with the exchange of A and B }
\end{gathered}
$$

We will see that the detailed expression of the tensor $\sigma_{\mathrm{ijpq}}$ does not matter here. The transition amplitude becomes

$$
\begin{aligned}
\Lambda_{\mathrm{i} \rightarrow \mathrm{f}}= & (2 \pi)^{-1}\left(\mathrm{t}_{\mathrm{f}}-\mathrm{t}_{\mathrm{i}}\right) \exp \left[-\mathrm{iE}_{0}\left(\mathrm{t}_{\mathrm{f}}-\mathrm{t}_{\mathrm{i}}\right) \hbar^{-1}\right] \int_{0}^{+\infty} \mathrm{d} \omega \sum_{\mathrm{ijklpq}} \sigma_{\mathrm{ijpq}}(\omega)_{\mathrm{B}} \alpha_{\mathrm{kl}}(\omega)_{\mathrm{A}} \\
& \left(-\mathrm{i} \nabla_{\mathrm{p}} \frac{1}{\mathrm{R}^{3}} \mathrm{D}_{\mathrm{ik}}(\mathrm{i} \omega \mathrm{R} / \mathrm{c})\right)\left(\mathrm{i} \nabla_{\mathrm{q}} \frac{1}{\mathrm{R}^{3}} \mathrm{D}_{\mathrm{jl}}(\mathrm{i} \omega \mathrm{R} / \mathrm{c})\right) \\
= & (2 \pi)^{-1}\left(\mathrm{t}_{\mathrm{f}}-\mathrm{t}_{\mathrm{i}}\right) \exp \left[-\mathrm{iE}_{0}\left(\mathrm{t}_{\mathrm{f}}-\mathrm{t}_{\mathrm{i}}\right) \hbar^{-1}\right] \int_{0}^{+\infty} \mathrm{d} \omega \sum_{\mathrm{ijklpq}} \sigma_{\mathrm{ijpq}}(\omega)_{\mathrm{B}} \alpha_{\mathrm{kl}}(\omega)_{\mathrm{A}} \\
& \mathrm{R}^{-8} \mathrm{~F}_{\mathrm{ikp}}(\mathrm{i} \omega \mathrm{R} / \mathrm{c}) \mathrm{F}_{\mathrm{jlq}}(\mathrm{i} \omega \mathrm{R} / \mathrm{c}) \\
+ & \text { a similar term with the exchange of A and B }
\end{aligned}
$$

which leads to an interaction energy of the following form:

$$
\begin{aligned}
\Delta \mathrm{E}(3)= & -(2 \pi)^{-1} \int_{0}^{+\infty} \hbar \mathrm{d} \xi \sum_{\mathrm{ijklpq}} \sigma_{\mathrm{ijpq}}(\mathrm{i} \xi)_{\mathrm{B}} \alpha_{\mathrm{kl}}(\mathrm{i} \xi)_{\mathrm{A}} \mathrm{R}^{-8} \mathrm{~F}_{\mathrm{ikp}}(-\xi \mathrm{R} / \mathrm{c}) \mathrm{F}_{\mathrm{jlq}}(-\xi \mathrm{R} / \mathrm{c}) \\
& + \text { a similar term with the exchange of A and B }
\end{aligned}
$$

\section{One M2 or E3 vortex}

In addition, at the same order of perturbation as just above, we have also to consider a fourth case where only one among the 4 interaction vortices is of magnetic quadrupole type (M2) or electric octupole type (E3), leading to change on that vortex d.e by $\mathrm{V}^{(\mathrm{M} 2)}=-\frac{1}{2} \nabla \mathbf{B}(\mathbf{R}): \mathbf{M}^{(2)}$ and $\mathrm{V}^{(\mathrm{E} 3)}=-\frac{1}{3 !} \nabla \nabla \mathbf{E}_{\perp}(\mathbf{R}): \mathbf{Q}^{(3)}$. In that case, equ.(2) has to be replaced by 
+ a similar term with the exchange of $\mathrm{A}$ and $\mathrm{B}$

As for $\sigma_{\mathrm{ijpq}}$, the exact expression of $\zeta_{\mathrm{jlpq}}$ does not matter. The transition amplitude becomes

$$
\begin{aligned}
\Lambda_{\mathrm{i} \rightarrow \mathrm{f}}= & (2 \pi)^{-1}\left(\mathrm{t}_{\mathrm{f}}-\mathrm{t}_{\mathrm{i}}\right) \exp \left[-\mathrm{iE}_{0}\left(\mathrm{t}_{\mathrm{f}}-\mathrm{t}_{\mathrm{i}}\right) \hbar^{-1}\right] \int_{0}^{+\infty} \mathrm{d} \omega \alpha_{\mathrm{ik}}(\omega)_{\mathrm{B}} \zeta_{\mathrm{jlpq}}(\omega)_{\mathrm{A}} \\
& \left(\frac{1}{\mathrm{R}^{3}} \mathrm{D}_{\mathrm{ik}}(\mathrm{i} \omega \mathrm{R} / \mathrm{c})\right)\left(-\nabla_{\mathrm{p}} \nabla_{\mathrm{q}} \frac{1}{\mathrm{R}^{3}} \mathrm{D}_{\mathrm{jl}}(\mathrm{i} \omega \mathrm{R} / \mathrm{c})\right) \\
= & (2 \pi)^{-1}\left(\mathrm{t}_{\mathrm{f}}-\mathrm{t}_{\mathrm{i}}\right) \exp \left[-\mathrm{iE}_{0}\left(\mathrm{t}_{\mathrm{f}}-\mathrm{t}_{\mathrm{i}}\right) \hbar^{-1}\right] \int_{0}^{+\infty} \mathrm{d} \omega \alpha_{\mathrm{ik}}(\omega)_{\mathrm{B}} \zeta_{\mathrm{jlpq}}(\omega)_{\mathrm{A}} \\
& \left(\frac{1}{\mathrm{R}^{3}} \mathrm{D}_{\mathrm{ik}}(\mathrm{i} \omega \mathrm{R} / \mathrm{c})\right)\left(\frac{1}{\mathrm{R}^{5}} \mathrm{G}_{\mathrm{jlpq}}(\mathrm{i} \omega \mathrm{R} / \mathrm{c})\right) \\
+ & \text { a similar term with the exchange of A and B }
\end{aligned}
$$

which leads to an interaction energy of the following form:

$$
\begin{aligned}
\Delta \mathrm{E}(4) & =-(2 \pi)^{-1} \int_{0}^{+\infty} \hbar \mathrm{d} \xi \sum_{\mathrm{j} j \mathrm{klpq}} \alpha_{\mathrm{ij}}(\mathrm{i} \xi)_{\mathrm{B}} \zeta_{\mathrm{klpq}}(\mathrm{i} \xi)_{\mathrm{A}} \mathrm{R}^{-8} \mathrm{D}_{\mathrm{ik}}(-\xi \mathrm{R} / \mathrm{c}) \mathrm{G}_{\mathrm{jlpq}}(-\xi \mathrm{R} / \mathrm{c}) \\
& + \text { a similar term with the exchange of A and B }
\end{aligned}
$$

\section{Consequences}

In summary, for uniformly distributed molecules we have identified in the previous section four terms $\Delta \mathrm{E}(1), \Delta \mathrm{E}(2), \Delta \mathrm{E}(3), \Delta \mathrm{E}(4)$ which can give a contribution to the interaction energy in addition to the Van der Waals term equ.(11). In the short distance limit where the retardation effects can be neglected $(\xi R / c<<1)$, the four expressions vary as $R^{-8}$ whereas the Van der Waals interaction varies as $\mathrm{R}^{-6}$. Assuming that the molecular states have neither well defined parity nor well defined angular momentum, all transitions are allowed and $a$ priori all four terms contribute with the same importance. Now $\Delta \mathrm{E}(3)$ and $\Delta \mathrm{E}(4)$ exist in all molecules and they are presumably much smaller than the Van der Waals interaction, and thus negligible. On the contrary $\Delta \mathrm{E}(1)$ and $\Delta \mathrm{E}(2)$, which involve the optical activity, only exist in chiral molecules. However we have seen that from symmetry arguments that the contribution of $\Delta \mathrm{E}(2)$ is presumably very weak with respect to $\Delta \mathrm{E}(1)$.

As a preliminary conclusion, if we consider a fluid of chiral molecules whose centres are supposed to be uniformly distributed, a possible orientation ordering will mainly result from two interactions, the anisotropic part of the Van der Waals coupling equ.(11), i.e. 
$-\int_{0}^{+\infty} \frac{\hbar}{\pi \mathrm{R}^{6}} \mathrm{~d} \xi\left(\alpha^{(2)}(\omega=\mathrm{i} \xi)_{\mathrm{B}} \cdot \alpha^{(2)}(\omega=\mathrm{i} \xi)_{\mathrm{A}}\right)$

and the chiral coupling $\Delta \mathrm{E}(1)$ as of equ.(36), i.e.

$\int_{0}^{+\infty} \frac{18 \hbar}{\pi \mathrm{R}^{8}} \mathrm{~d} \xi\left(\eta^{(2)}(\omega=\mathrm{i} \xi)_{\mathrm{B}} \cdot \eta^{(2)}(\omega=\mathrm{i} \xi)_{\mathrm{A}}\right)$

Both interactions have different ranges (the dipolar term varies like $\mathrm{R}^{-6}$ and the chiral term varies like $\mathrm{R}^{-8}$ ) and different symmetries; both have the same behaviour through rotation but not through reflection. It is well known that a system undergoing forces with different ranges and symmetries may minimise its free energy by adopting an helical ordering; it is the case of the helimagnetic configuration of some ferro-magnets or of the twisted configuration of the chiral nematics (cholesterics) [14].

\section{Nonlinear terms}

It is worth underlining that we have only considered linear couplings, i.e. involving only two virtual photons. We could also think of nonlinear couplings involving three or more virtual photons. For instance, a three-photon coupling with only electric dipole transitions, as it has been considered by Harris [15], if allowed by the molecular symmetries, would also brings in a chiral interaction, and its dynamic expression would naturally introduce the first nonlinear polarisability $\beta_{\mathrm{ijk}}\left(\omega_{1}, \omega_{2}, \omega_{3}\right)$ with $\omega_{1}+\omega_{2}+\omega_{3}=0$, responsible for the parametric optical phenomena at molecular level. $\beta$ is a rank-3 true tensor, null for centro-symmetric molecules In terms of irreducible tensor components with respect to rotations and index permutations (see Appendix A2), $\beta$ has the following spectrum:

$$
\{3\}\left(\beta^{(3)}+\beta^{(1)}\right),\{21\}\left(\beta^{\prime(1)}+\beta^{\prime \prime(1)}+\beta^{\prime(2)}+\beta^{\prime \prime(2)}\right),\left\{1^{3}\right\} \beta^{(0)}
$$

In an isotropic medium, it would lead to an intermolecular coupling of the following form:

$$
\Delta \mathrm{E} \propto \int_{0}^{+\infty} \int_{0}^{+\infty} \mathrm{d} \xi_{1} \mathrm{~d} \xi_{2} \sum_{\mathrm{J}=0,1,2,3}\left(\beta^{(\mathrm{J})}\left(\omega_{1}=\mathrm{i} \xi_{1}, \omega_{2}=\mathrm{i} \xi_{2}\right)_{\mathrm{B}} \cdot \beta^{(\mathrm{J})}\left(\omega_{1}=\mathrm{i} \xi_{1}, \omega_{2}=\mathrm{i} \xi_{2}\right)_{\mathrm{A}}\right) \mathrm{R}^{-9} \Phi\left(\xi_{1} \mathrm{R} / \mathrm{c}, \xi_{2} \mathrm{R} / \mathrm{c}\right)
$$

In the quasi-static limit, the contribution of the pseudo-scalar component $\beta^{(0)}$ is the term proposed by Schipper $[16,17]$ as able to account for chiral discrimination. As far as the Rdependence is concerned, the leading term in equ.(40) decreases with distance as $\mathrm{R}^{-9}$, faster that the chiral term equ.(38) so that it will not be further considered in the present paper. However, that nonlinear term and the chiral term equ.(38) have in common to originate from third-order dispersion interactions.

\section{Static terms}

In addition we have only considered so far the dynamic interactions due to the transverse electromagnetic field but not the static interactions due to the longitudinal electric field. .The general expression of those static interactions is 


$$
\mathrm{U}_{\mathrm{AB}}=\sum_{\mathrm{j}_{\mathrm{A}}, \mathrm{j}_{\mathrm{B}}}\left(\left\{\mathfrak{M}^{\left(\mathrm{j}_{\mathrm{A}}\right)} \otimes \mathfrak{M}^{\left(\mathrm{j}_{\mathrm{B}}\right)}\right\}^{\left(\mathrm{j}_{\mathrm{A}}+\mathrm{j}_{\mathrm{B}}\right)} \cdot \frac{\mathrm{C}^{\left(\mathrm{j}_{\mathrm{A}}+\mathrm{j}_{\mathrm{B}}\right)}\left(\mathbf{R}_{\mathrm{AB}} / \mathrm{R}\right)}{\mathrm{R}^{\mathrm{j}_{\mathrm{A}}+\mathrm{j}_{\mathrm{B}}+1}}\right)
$$

where $\mathfrak{M}^{(\mathrm{j})}$ is an electric multipole, irreducible tensor of order $\mathrm{j}$ and $\mathrm{C}_{\mathrm{M}}^{(\mathrm{J})}(\theta, \varphi)$ is the unit spherical tensor of rank $\mathbf{J}$ (see Appendix A3). They have a null contribution through a geometrical average [18] which allows discarding them inasmuch as we restrict ourselves to the case of uncorrelated molecules whose centres are supposed to be randomly distributed. We will come back to the role of those terms in the last section.

\section{Nematic order}

Let us choose the main axes of $\alpha^{(2)}{ }_{\mathrm{A}}$ and $\alpha^{(2)}{ }_{\mathrm{B}}$ as the co-ordinate axes of the molecular frames of the molecules $\mathrm{A}$ and $\mathrm{B}$ respectively. The expression of $\Delta \mathrm{E}$ as given by equ.(11) behaves in a rotation like

$$
\Delta \mathrm{E}=-\mathrm{H}_{0}(\mathrm{R})-\mathrm{H}_{2}(\mathrm{R})\left(\mathrm{C}^{(2)}\left(\theta_{\mathrm{A}}, \varphi_{\mathrm{A}}\right) \cdot \mathrm{C}^{(2)}\left(\theta_{\mathrm{B}}, \varphi_{\mathrm{B}}\right)\right)
$$

Let us note as $<>$ an averaging over the molecular orientations and let us take the main axes of $\left\langle\alpha^{(2)}>\right.$ as the co-ordinate axes of the macroscopic frame $\mathrm{XYZ}$, hence $<\alpha_{\mathrm{XY}}>=<\alpha_{\mathrm{YZ}}>=<\alpha_{\mathrm{ZX}}>=0$, or $\left.\left.\left\langle\mathrm{C}_{1}^{(2)}\right\rangle=<\mathrm{C}_{-1}^{(2)}\right\rangle=<\mathrm{C}_{2}^{(2)}-\mathrm{C}_{-2}^{(2)}\right\rangle=0$. Assuming an axial symmetry around the macroscopic $\mathrm{Z}$ axis gives $\left\langle\mathrm{C}_{2}^{(2)}+\mathrm{C}_{-2}^{(2)}\right\rangle \propto\left\langle\sin ^{2} \theta \cos 2 \varphi\right\rangle=0$, so that the only non vanishing component of $\left\langle\mathrm{C}_{\mathrm{Q}}^{(2)}\right\rangle$ is $\left\langle\mathrm{C}_{0}^{(2)}\right\rangle=\left\langle\mathrm{P}_{2}(\cos \theta)\right\rangle=\left\langle\frac{3 \cos ^{2} \theta-1}{2}\right\rangle$.

In the molecular field approach of the isotropic - nematic phase transition of non chiral molecules, first elaborated by Maier and Saupe [19, 20], the phase transition is a first order one with order parameter $\mathrm{p}=\left\langle\mathrm{P}_{2}(\cos \theta)\right\rangle$. $\mathrm{p}$ is the solution of a self-consistent equation of the following form:

$$
\mathrm{p}=\frac{\int \mathrm{d} \Omega \mathrm{P}_{2}(\cos \theta) \exp \left[-\mathrm{JpP}_{2}(\cos \theta) / \mathrm{k}_{\mathrm{B}} \mathrm{T}\right]}{\int \mathrm{d} \Omega \exp \left[-\mathrm{JpP}_{2}(\cos \theta) / \mathrm{k}_{\mathrm{B}} \mathrm{T}\right]}
$$

where $\mathbf{J}$ is an effective coupling constant. If $\mathrm{J}>0$, that equation only has the trivial solution $\mathrm{p}=0$. If $\mathrm{J}<0$, depending on the temperature, the molecules may adopt an orientation ordering; in the ordered phase (nematic) $1 \geq p>0$, in the disordered phase (isotropic) $p=0$.

\section{Helical ordering of chiral nematics}

The short range molecular interaction in the electric dipole approximation can be accounted for in a quasi-static model by an induced electric dipole - induced electric dipole interaction, which actually is the static limit of the full quantum electrodynamics calculation as of equ.(37). Goossens [21] and Van der Meer et al. [22] had extended that quasi-static approach by introducing an additional induced electric dipole - induced electric quadrupole coupling; the isotropic - nematic phase transition described above is complemented by the prediction of 
a helical pitch for optically active nematics. However, the expression that they retain for the chiral term should actually give a null contribution after averaging over molecular positions if the centres of gravity of the molecules are uncorrelated and isotropically distributed. That additional term of which the effective dependence upon the inter-molecular distance is in $\mathrm{R}^{-}$ ${ }^{7} ;$,is the static limit of the chiral term of equs.(15-20).

Comparing the quasi-static models with the present approach of dynamic molecular interactions, it is worth noticing that the electric quadrupole part of the optical activity contributes to the irreducible components of weight $\mathrm{J}=1,2,3$ of the optical activity whereas the magnetic dipole part of $\eta$ gives contributions of weight $J=0,1,2$. Hence $\eta^{(2)}$ contains contributions of both E2 and M1 type.

It has also to be noticed that in the present work no assumption is made upon the shape of the molecules, neither an effective cylindrical symmetry, i.e. the molecules are assumed to be free to rotate around a molecular axis, ,nor a planar like geometry. Those assumptions, which have been made in earlier works for the sake of computational simplicity, are actually incompatible with chirality [23].

If we now choose the main axes of $\eta^{(2)}$ and $\eta^{(2)}{ }_{B}$ as the co-ordinate axes of the molecular frames for the molecules A and B respectively (a priori the main axes of the molecular tensors $\alpha^{(2)}$ and $\eta^{(2)}$ do not coincide) and if $\rho^{\prime}$ is the angle between the axes $Z^{\prime}{ }_{\mathrm{A}}$ and $Z^{\prime}{ }_{\mathrm{B}}$, $\Delta \mathrm{E}(1)$ behaves in a rotation as

$$
\Delta \mathrm{E}^{\mathrm{iso}}(1)=\mathrm{X}_{2}(\mathrm{R})\left(\mathrm{C}^{(2)}\left(\theta_{\mathrm{A}}^{\prime}, \varphi_{\mathrm{A}}^{\prime}\right) \cdot \mathrm{C}^{(2)}\left(\theta_{\mathrm{B}}^{\prime}, \varphi_{\mathrm{B}}^{\prime}\right)\right)
$$

Now, $\rho$ being the angle between the axes $Z_{A}$ and $Z_{B}$

$\left(\mathrm{C}^{(2)}\left(\theta_{\mathrm{A}}, \varphi_{\mathrm{A}}\right) \cdot \mathrm{C}^{(2)}\left(\theta_{\mathrm{B}}, \varphi_{\mathrm{B}}\right)\right)=\mathrm{P}_{2}(\cos \rho)$

and $\rho$ ' be the angle between the axes $Z^{\prime}{ }_{\mathrm{A}}$ and $Z^{\prime}{ }_{\mathrm{B}}$

$\left(C^{(2)}\left(\theta^{\prime}{ }_{A}, \varphi^{\prime}{ }_{A}\right) \cdot C^{(2)}\left(\theta^{\prime}{ }_{B}, \varphi^{\prime}{ }_{B}\right)\right)=P_{2}\left(\cos \rho^{\prime}\right)$

where $P_{K}$ is the Legendre polynomial of order $K$ (see Appendix A3).

From equs.(42) \& (44), the total interaction energy of the pair A, B appears to behave as

$$
\mathrm{U}_{\mathrm{AB}}(\mathrm{R})=-\mathrm{H}_{0}(\mathrm{R})-\mathrm{H}_{2}(\mathrm{R}) \mathrm{P}_{2}(\cos \rho)+\mathrm{X}_{2}(\mathrm{R}) \mathrm{P}_{2}\left(\cos \rho^{\prime}\right)
$$

The scalar product of the two unit tensors does not depend upon the reference frame, hence $\rho=\rho^{\prime}$.

The dipolar interaction (the anisotropic part of the Van der Waals forces) and the chiral interaction possess the same properties as far as the relative molecular orientations are concerned. Now, the dipolar interaction is always attractive and tends to align the molecules parallel or anti-parallel to one of the main axes of $\alpha^{(2)}$. Conversely the chiral term is always repulsive and tends to align the molecules perpendicular to one of the main axes of $\eta^{(2)}$.

Moreover they have different ranges although the dipolar coupling is likely to be more intense than the chiral coupling.

A Maier - Saupe - type mean field approach, based on the relative molecular orientations independently of the molecular positions, will account for an isotropic - nematic phase transition as above, but not for the possibility of a cholesteric phase whose helical ordering 
implies a correlation between position and orientation. We thus have to tackle the problem differently; the simple model below accounts for the possibility of helical ordering.

The molecular axes $Z_{A}$ and $Z^{\prime}{ }_{A}\left(\operatorname{resp} . Z_{B}\right.$ and $\left.Z^{\prime}{ }_{B}\right)$ determine a plan $\Pi_{A}\left(\operatorname{resp} . \Pi_{B}\right) . \Pi_{A}$ and $\Pi_{B}$ intersect each other along a direction which we choose as the $Z$ axis. Let $\theta_{A}\left(\operatorname{resp} \theta_{B}\right)$ be the angle between $\mathrm{Z}$ and $\mathrm{Z}_{\mathrm{A}}$ (resp. $\mathrm{Z}$ and $\mathrm{Z}_{\mathrm{B}}$ ), let $\lambda$ be the angle between $\mathrm{Z}_{\mathrm{A}}$ and $\mathrm{Z}^{\prime}{ }_{\mathrm{A}}$ ( or $\mathrm{Z}_{\mathrm{B}}$ and $Z^{\prime}{ }_{B}$ ) and let $\Phi$ be the angle between $\Pi_{A}$ and $\Pi_{B}$ (see fig. 2).

One has:

$$
\begin{aligned}
& \cos \rho=\cos \left(Z_{A} Z_{B}\right)=\sin \theta_{A} \sin \theta_{B} \cos \Phi+\cos \theta_{A} \cos \theta_{B} \\
& \cos \rho^{\prime}=\cos \left(Z_{A}^{\prime} Z_{B}^{\prime}\right)=\sin \left(\theta_{A}+\lambda\right) \sin \left(\theta_{B}+\lambda\right) \cos \Phi+\cos \left(\theta_{A}+\lambda\right) \cos \left(\theta_{B}+\lambda\right)
\end{aligned}
$$

Introducing equ.(47) into equ.(46) and writing $\left(\frac{\partial U}{\partial \Phi}\right)_{R}=0$, we obtain the minima of $U$ with respect to $\Phi$ for any given value of R. We find two solutions:

$\Phi=0, \theta_{\mathrm{A}}=\theta_{\mathrm{B}}$, i.e. a parallel configuration, and

$\Phi=\pi, \theta_{\mathrm{B}}=\pi-\theta_{\mathrm{A}}$, i.e. an anti-parallel configuration.

In both cases, $\Pi_{A}$ and $\Pi_{B}$ are identical, i.e. the molecules are in the same plane, with a nematic-type ordering.

The $\mathrm{Z}$ axis as defined above (intersection of $\Pi_{\mathrm{A}}$ and $\Pi_{\mathrm{B}}$ ) does not exist if the molecules are in parallel planes. In that case we adopt a different approach. Let $\mathrm{Z}$ now be the axis perpendicular to $\Pi_{\mathrm{A}}$ and $\Pi_{\mathrm{B}}$ and let $\theta$ be the angle between $Z$ and $\mathbf{R}$ (see fig. 3). We look for the possibility of helical ordering by putting

$$
\mathrm{t}=\cos \rho, \rho=q Z, Z=R \cos \theta
$$

At short distance, the molecules, which are supposed to be able to rotate freely and whose centres are assumed to be randomly distributed, feel a strong repulsive interaction due to the exclusion principle. In that section, that will be accounted for by an effective hard-core intermolecular potential of radius D. We will express the dipolar interaction as

$$
\mathrm{H}_{2}(\mathrm{R})=\mathrm{h}\left(\frac{\mathrm{D}}{\mathrm{R}}\right)^{6} \text { for } \mathrm{R}>\mathrm{D}
$$

where $\mathrm{h}$ is a function of the squared norms of the quadrupolar parts of the molecular polarisabilities, i.e. $\left(2 \alpha_{z z}-\alpha_{x x}-\alpha_{y y}\right)^{2}$, and in a similar way we will express the chiral interaction as

$$
\mathrm{X}_{2}(\mathrm{R})=\chi\left(\frac{\mathrm{D}}{\mathrm{R}}\right)^{8} \text { for } \mathrm{R}>\mathrm{D}
$$

where $\chi$ is a function of to the squared norms of the quadrupolar parts of the optical activities, i.e. $\left(2 \eta_{\mathrm{zyx}}-\eta_{\mathrm{xyz}}-\eta_{\mathrm{zxy}}\right)^{2}$.

The average interaction energy of 2 molecules A and B can thus be written as 


$$
\begin{aligned}
\left\langle\mathrm{U}_{\mathrm{AB}}\right\rangle & =\int \frac{d^{3} \mathbf{R}_{\mathrm{AB}}}{\mathrm{V}} \mathrm{Y}(\mathrm{R}-\mathrm{D}) \mathrm{U}_{\mathrm{AB}}(\mathrm{R}) \\
& =\left\langle-\mathrm{H}_{0}\right\rangle+\int \frac{\mathrm{R}^{2} d \mathrm{R}}{\mathrm{V}} \mathrm{Y}(\mathrm{R}-\mathrm{D})\left[-\mathrm{H}_{2}(\mathrm{R})+\mathrm{X}_{2}(\mathrm{R})\right] \int 2 \pi \sin \theta d \theta \mathrm{P}_{2}(\cos \rho)
\end{aligned}
$$

where $\mathrm{Y}(\mathrm{R}-\mathrm{D})=0$ if $\mathrm{R}<\mathrm{D}$ and $\mathrm{Y}(\mathrm{R}-\mathrm{D})=1$ if $\mathrm{R}>\mathrm{D}$. Writing

$$
\mathrm{P}_{2}(\cos \rho)=\frac{3 \cos ^{2} \rho-1}{2}=\frac{1}{4}+\frac{3}{4} \cos 2 \rho=\frac{1}{4}+\frac{3}{4} \operatorname{Re}(\exp 2 \mathrm{iqR} \cos \theta)
$$

we have

$$
\left\langle\mathrm{U}_{\mathrm{AB}}\right\rangle=\left\langle-\mathrm{H}_{0}\right\rangle+\pi \int_{\mathrm{D}}^{\infty} \frac{\mathrm{R}^{2} d \mathrm{R}}{\mathrm{V}}\left[-\mathrm{H}_{2}(\mathrm{R})+\mathrm{X}_{2}(\mathrm{R})\right]\left(1+3 \frac{\sin (2 \mathrm{qR})}{(2 \mathrm{qR})}\right) .
$$

The minimum of $\left\langle U_{A B}\right\rangle$ is obtained from $\frac{\partial\left\langle U_{A B}\right\rangle}{\partial q}=0$, i.e.

$$
0=\int_{D}^{\infty} R^{3} d R\left[-H_{2}(R)+X_{2}(R)\right]\left(\frac{z \cos z-\sin z}{z^{2}}\right)_{z=2 q R} .
$$

i.e. either $\mathrm{z}=0$ or

$$
0=\int_{2 \mathrm{qD}}^{\infty} d \mathrm{z}\left[-\mathrm{h}\left(\frac{\mathrm{z} \cos \mathrm{z}-\sin \mathrm{z}}{\mathrm{z}^{5}}\right)+\chi(2 \mathrm{qD})^{2}\left(\frac{\mathrm{z} \cos \mathrm{z}-\sin \mathrm{z}}{\mathrm{z}^{7}}\right)\right] .
$$

The first integral in equ.(54) is

$$
-\frac{h}{8}\left[\frac{1}{z^{4}}\left(2 \sin z-2 z \cos z+z^{2} \sin z+z^{3} \cos z\right)\right]_{2 q D}^{\infty}-\frac{h}{8} \int_{2 q D}^{\infty} d z \frac{\sin z}{z},
$$

and the second one is

$$
\frac{\chi(2 q D)^{2}}{144}\left[\frac{1}{z^{6}}\left(24 \sin z-24 z \cos z+6 z^{2} \sin z+2 z^{3} \cos z-z^{4} \sin z-z^{5} \cos z\right)\right]_{2 q D}^{\infty}-\frac{\chi(2 q D)^{2}}{144} \int_{2 q D}^{\infty} d z \frac{\sin z}{z}
$$

That allows to determine $\mathrm{q}$ as a function of $\mathrm{h}, \chi, \mathrm{D}$, and hence the pitch $\mathrm{L}$ of the helix as $\mathrm{L}=2 \pi / \mathrm{q}$. Assuming $\mathrm{L}>>\mathrm{D}$, i.e. $2 \mathrm{qD}<<1$, we get

$$
0 \simeq \mathrm{h}\left[\frac{1}{3 \mathrm{z}}-\frac{11 \mathrm{z}}{120}-\frac{1}{8}\left(\frac{\pi}{2}-\mathrm{z}\right)+\mathrm{o}\left(\mathrm{z}^{2}\right)\right]_{\mathrm{z}=2 \mathrm{qD}}+\chi\left[\frac{-1}{9 \mathrm{z}}+\frac{\mathrm{z}}{30}+\mathrm{o}\left(\mathrm{z}^{2}\right)\right]_{\mathrm{z}=2 \mathrm{qD}}
$$

i.e.

$$
\frac{1}{30}(\mathrm{~h}+\chi) \mathrm{z}-\left(\frac{\pi \mathrm{h}}{16}\right)+\frac{1}{3 \mathrm{z}}\left(\mathrm{h}-\frac{\chi}{3}\right) \simeq 0
$$

If $\mathrm{h}^{2}\left(\left(\frac{\pi \mathrm{h}}{16}\right)^{2}-\frac{2}{45}\right)-\frac{4}{135} \mathrm{~h} \chi+\frac{2}{135} \chi^{2}<0$, equ.(56b) has no roots, and equ.(53) has only the trivial solution $\mathrm{z}=0$, i.e. $\mathrm{L} \rightarrow \infty$ (nematic ordering). 


\section{Molecular correlations}

Our result is not fully satisfactory since the pitch as derived above is temperatureindependent, which is in obvious disagreement with the observations. In order to account for a temperature dependence of the pitch, we could think of adding other interaction terms, with a different behaviour with respect to rotational symmetries. In the present work we have indeed neglected terms that would produce a dependence in $\mathrm{P}_{4}(\cos \rho)$. The consideration of those terms in addition to the only dependence in $\mathrm{P}_{2}(\cos \rho)$ that we have previously retained would produce a temperature dependent pitch, as it has been pointed out by Lin-Liu et al. [24].

However, those neglected terms are presumably very small, and other explanations are likely to be looked for. It is alternatively plausible that the anisotropic contributions to the short range repulsive forces due to the exclusion principle play a significant role, and that the effective spherical hard-core is not a valid assumption for taking the steric effects into account.

Let $\mathrm{g}(\mathrm{A}, \mathrm{B})$ be the pair correlation function of the two molecules $\mathrm{A}$ and $\mathrm{B}$, and $\mathrm{g}_{\mathrm{HC}}(\mathrm{A}, \mathrm{B})$ the pair correlation function due to the short range exclusion principle driven forces alone. The repulsion forces will be approximated by an anisotropic hard-core potential and the electromagnetic interactions $\mathrm{U}_{\mathrm{AB}}$ (static and dynamic) will be treated as a perturbation:

$$
\mathrm{g}(\mathrm{A}, \mathrm{B}) \simeq \mathrm{g}_{\mathrm{HC}}(\mathrm{A}, \mathrm{B}) \exp \left(-\frac{\mathrm{U}_{\mathrm{AB}}}{\mathrm{k}_{\mathrm{B}} \mathrm{T}}\right) \simeq \mathrm{g}_{\mathrm{HC}}(\mathrm{A}, \mathrm{B})\left(1-\frac{\mathrm{U}_{\mathrm{AB}}}{\mathrm{k}_{\mathrm{B}} \mathrm{T}}\right)
$$

$\mathrm{g}_{\mathrm{HC}}(\mathrm{A}, \mathrm{B})$ depends on density and geometry but not explicitly on temperature. It is a function of the intermolecular distance $\mathrm{R}$ and of the mutual orientation of the molecules. That orientation can be expressed by two angles, the angle $\rho$ of the rotation which has the $Z_{A}$ and $Z_{B}$ axes coincide and the angle $\varphi$ of the following rotation which has the $X_{A}, Y_{A}$ and $X_{B}, Y_{B}$ axes coincide. $\mathrm{g}_{\mathrm{HC}}(\mathrm{A}, \mathrm{B})$ can quite generally be expanded as a series of spherical harmonics: $\mathrm{g}_{\mathrm{HC}}(\mathrm{A}, \mathrm{B})=\sum_{\mathrm{K}, \mathrm{Q}} \mathrm{g}_{\mathrm{Q}}^{(\mathrm{K})}(\mathrm{R}) \mathrm{C}_{\mathrm{Q}}^{(\mathrm{K})}(\rho, \varphi)$. 
with

$\mathrm{g}_{\mathrm{Q}}^{(\mathrm{K})}(\mathrm{R})=\frac{2 \mathrm{~K}+1}{4 \pi} \iint \sin \rho d \rho d \varphi \mathrm{C}_{\mathrm{Q}}^{(\mathrm{K})^{*}}(\rho, \varphi) \mathrm{g}_{\mathrm{HC}}(\mathrm{R}, \rho, \varphi)$

Now, $\mathrm{g}_{\mathrm{HC}}(\mathrm{A}, \mathrm{B})$ is a true scalar (parity +1 ) whereas the $\mathrm{C}_{\mathrm{Q}}^{(\mathrm{K})}$ have the parity $(-1)^{\mathrm{K}}$.

Consequently the terms with odd values of $\mathrm{K}$ are null. It is worth noticing there is no need to make assumptions on the uniaxial or biaxial character of the correlations [25, 26, 27].

The calculation of the average intermolecular energy equ.(50) becomes

$$
\left\langle\mathrm{U}_{\mathrm{AB}}\right\rangle \simeq \int \frac{d^{3} \mathbf{R}}{\mathrm{V}} \mathrm{g}_{\mathrm{HC}}(\mathrm{A}, \mathrm{B})\left(1-\frac{\mathrm{U}_{\mathrm{AB}}}{\mathrm{k}_{\mathrm{B}} \mathrm{T}}\right) \mathrm{U}_{\mathrm{AB}}
$$

We first perform the integration over $\varphi$ :

$\int d \varphi \mathrm{C}_{\mathrm{Q}}^{(\mathrm{K})}(\rho, \varphi)=2 \pi \delta_{\mathrm{Q} 0} \mathrm{P}_{\mathrm{K}}(\cos \rho)$

where $\mathrm{g}_{\mathrm{HC}}(\mathrm{A}, \mathrm{B})$ is given by equ.(60) and $\mathrm{U}_{\mathrm{AB}}$ is given by equ.(46); only the $\mathrm{Q}=0$ terms are relevant, hence we get

$$
\left\langle\mathrm{U}_{\mathrm{AB}}\right\rangle=\left\langle-\mathrm{U}_{0}\right\rangle+\sum_{\text {Keven }} \int \frac{\mathrm{R}^{2} d \mathrm{R}}{\mathrm{V}} \mathrm{g}_{0}^{(\mathrm{K})}(\mathrm{R}) \int 2 \pi \sin \rho d \rho \mathrm{P}_{2}(\cos \rho) \mathrm{U}_{\mathrm{AB}}
$$

The isotropic component $\mathrm{g}_{0}^{(0)}(\mathrm{R})$ is the usual radial pair correlation function for hard spheres. It is null if $\mathrm{R}$ is less than some effective molecular diameter $\mathrm{D}$ and it tends toward 1 after a few rapidly decreasing oscillations over a distance of a few $\mathrm{D}$. The anisotropic components $\mathrm{g}_{0}^{(\mathrm{K} \neq 0)}(\mathrm{R})$ are null if $\mathrm{R}$ is less than some minimum distance and they tend rapidly toward 0 over a distance of a few $\mathrm{D}$.

Equ.(63) will then produce several types of contributions:

(1) An isotropic one, essentially coming from the association of $g_{0}^{(0)}(R)$ with the isotropic part of $\mathrm{U}_{\mathrm{AB}}$, essentially the Van der Waals potential.

(2) One coming from the association of $g_{0}^{(0)}(R)$ with the anisotropic part of $U_{A B}$. The calculation is qualitatively similar as it was in the previous section (and identical in a mean field description of fluids where $\mathrm{g}_{0}^{(0)}(\mathrm{R})=0$ for $\mathrm{R}<\mathrm{D}, \mathrm{g}_{0}^{(0)}(\mathrm{R})=1$ for $\left.\mathrm{R}>\mathrm{D}\right)$ ). It accounts for a temperature independent helical pitch.

(3) One coming from the association of $g_{0}^{(K \neq 0)}(R) P_{K}(\cos \rho)$ with the isotropic part of $U_{A B}$. That term exists in all molecules (as $\mathrm{K}$ shall be even) and it is thus superimposed to the anisotropic Van der Waals forces. The principle of the calculation is the same as in the previous section and results in a temperature independent helical pitch. A priori, the isotropic and the anisotropic parts of the Van der Waals forces have the same order of magnitude for very a-spherical molecules. However the $\mathrm{g}_{0}^{(\mathrm{K} \neq 0)}$, which are exactly null in the mean field model of liquids, are presumably weak if the molecules are supposed to be free to rotate, as it is the case in the isotropic and in the nematic phases. Consequently that term is supposed to be smaller than the term (2).

(4) One coming from $g_{0}^{(\mathrm{K})} \mathrm{U}_{\mathrm{AB}} \mathrm{U}_{\mathrm{AB}} / \mathrm{k}_{\mathrm{B}} \mathrm{T}$. It needs more attention. If $\mathrm{U}_{\mathrm{AB}}$ is the dynamic interaction given by equ.(43), it will produce terms in $\mathrm{P}_{\mathrm{L}}(\cos \rho)$ with $\mathrm{L}=0,2,4,6,8$. The 
addition of those terms would result in a dependence of the pitch with temperature but they are presumably very weak because of their R-dependence in $\mathrm{R}^{-12}$ or $\mathrm{R}^{-14}$ or $\mathrm{R}^{-16}$.

However, up to now, we have only considered the dynamic interactions due to exchange of virtual transverse photons but not the static interactions due to the longitudinal electric field. We have already seen that those static interactions, whose general expression is given by equ.(39) above have a null contribution through a geometrical average if the centres of the molecules are randomly distributed and if correlations are neglected.

We will retain as the dominant terms the permanent electric dipole - permanent electric dipole interaction, the permanent electric dipole - permanent electric quadrupole interaction and the permanent electric quadrupole - permanent electric quadrupole interaction.

The association of either of those interactions with $\mathrm{g}_{0}^{(\mathrm{K} \neq 0)}(\mathrm{R}) \mathrm{P}_{\mathrm{K}}(\cos \rho)$ gives no scalar contribution (as $\mathrm{K}$ shall be even) but the thermodynamic correlations expressed by equ.(61) allow them to provide a non zero scalar contribution. Indeed, the square of each of those two expressions gives a scalar term after some re-arrangements (their product does not). We thus find as dominant terms 3 contributions involving the scalar product of even multipoles

- from the permanent electric dipole - permanent electric dipole interaction

$$
\left\langle\mathrm{U}_{\mathrm{AB}}\right\rangle=\frac{-1}{\mathrm{k}_{\mathrm{B}} \mathrm{T}} \int \frac{d^{3} \mathbf{R}_{\mathrm{AB}}}{\mathrm{V}} \mathrm{g}_{0}^{(0)}(\mathrm{R}) \frac{1}{\mathrm{R}^{6}}\left(4 \pi \epsilon_{0}\right)^{-2} \sum_{\mathrm{j}=0,2}\left(\left\{\mu_{\mathrm{A}} \otimes \mu_{\mathrm{A}}\right\}^{(\mathrm{j})} \cdot\left\{\mu_{\mathrm{B}} \otimes \mu_{\mathrm{B}}\right\}^{(\mathrm{j})}\right)
$$

- from the permanent electric dipole - permanent electric quadrupole interaction

$$
\begin{aligned}
\left\langle\mathrm{U}_{\mathrm{AB}}\right\rangle= & \frac{1}{\mathrm{k}_{\mathrm{B}} \mathrm{T}} \int \frac{d^{3} \mathbf{R}_{\mathrm{AB}}}{\mathrm{V}} \mathrm{g}_{0}^{(0)}(\mathrm{R}) \frac{1}{\mathrm{R}^{8}}\left(4 \pi \epsilon_{0}\right)^{-2} \sum_{\mathrm{j}=0,2}\left(\left\{\mu_{\mathrm{A}} \otimes \mu_{\mathrm{A}}\right\}^{(\mathrm{j})} \cdot\left\{\mathrm{Q}_{\mathrm{B}} \otimes \mathrm{Q}_{\mathrm{B}}\right\}^{(\mathrm{j})}\right) \\
& + \text { a similar term with the exchange of } \mathrm{A} \text { and } \mathrm{B}
\end{aligned}
$$

- from the permanent electric quadrupole - permanent electric quadrupole interaction

$$
\left\langle\mathrm{U}_{\mathrm{AB}}\right\rangle=\frac{-1}{\mathrm{k}_{\mathrm{B}} \mathrm{T}} \int \frac{d^{3} \mathbf{R}_{\mathrm{AB}}}{\mathrm{V}} \mathrm{g}_{0}^{(0)}(\mathrm{R}) \frac{1}{\mathrm{R}^{10}}\left(4 \pi \epsilon_{0}\right)^{-2} \sum_{\mathrm{j}=0,2,4}\left(\left\{\mathrm{Q}_{\mathrm{A}} \otimes \mathrm{Q}_{\mathrm{A}}\right\}^{(\mathrm{j})} \cdot\left\{\mathrm{Q}_{\mathrm{B}} \otimes \mathrm{Q}_{\mathrm{B}}\right\}^{(\mathrm{j})}\right)
$$

and one contribution from the permanent electric dipole - permanent electric quadrupole interaction involving the scalar product of even and odd multipoles

$$
\left\langle\mathrm{U}_{\mathrm{AB}}\right\rangle=\frac{-2}{\mathrm{k}_{\mathrm{B}} \mathrm{T}} \int \frac{d^{3} \mathbf{R}_{\mathrm{AB}}}{\mathrm{V}} \mathrm{g}_{0}^{(0)}(\mathrm{R}) \frac{1}{\mathrm{R}^{8}}\left(4 \pi \epsilon_{0}\right)^{-2} \sum_{\mathrm{j}=1,2,3}\left(\left\{\mu_{\mathrm{A}} \otimes \mathrm{Q}_{\mathrm{A}}\right\}^{(\mathrm{j})} \cdot\left\{\mu_{\mathrm{B}} \otimes \mathrm{Q}_{\mathrm{B}}\right\}^{(\mathrm{j})}\right)
$$

$\mu$ being a true vector and $\mathrm{Q}$ a true quadrupole (i.e. a symmetric traceless rank 2 tensor), the rank 3 tensor $\left\{\mu_{\mathrm{A}} \otimes \mathrm{Q}_{\mathrm{A}}\right\}^{(\mathrm{j})}$ has the following spectrum in terms of irreducible tensor components with respect to rotations and index permutations (see Appendix A2): $\{3\}\left\{\mu_{\mathrm{A}} \otimes \mathrm{Q}_{\mathrm{A}}\right\}^{(3)},\{21\}\left\{\mu_{\mathrm{A}} \otimes \mathrm{Q}_{\mathrm{A}}\right\}^{(2)},\{21\}\left\{\mu_{\mathrm{A}} \otimes \mathrm{Q}_{\mathrm{A}}\right\}^{(1)}$. In particular, it has a pseudo quadrupolar component $\mathrm{j}=2$, which is present in chiral molecules.

A priori the term equ.(64b) is smaller than the term equ.(64a), although it has the same symmetries, and the term equ.(64c) is still smaller. The terms equ.(64b) and equ.(64c) should be of the same order of magnitude.

Now, let us consider more closely the terms $j=2$ in equ.(64a) and equ.(64d). They have respectively the same R-dependence as the two dynamic interactions we have considered so far, the coupling of the polarisabilities $\left(\mathrm{R}^{-6}\right)$ and the coupling of the optical activities $\left(\mathrm{R}^{-8}\right)$. In 
addition whereas the $\mathrm{j}=2$ contribution in equ.(64a) is the scalar product of true quadrupoles (like the anisotropic Van der Waals coupling), the $\mathrm{j}=2$ contribution in equ.(64d) is the scalar product of pseudo quadrupoles (like the coupling of the optical activities).

As a consequence, those two terms can be embedded in the parameters $h$ and $\chi$ respectively introduced in equs.(49a) \& (49b) which will then be replaced by effective, temperature dependent, parameters $\mathrm{h}^{*}=\mathrm{h}\left(1-\frac{\mathrm{J}_{\mathrm{h}}}{\mathrm{k}_{\mathrm{B}} \mathrm{T}}\right)$ and $\chi^{*}=\chi\left(1-\frac{\mathrm{J}_{\chi}}{\mathrm{k}_{\mathrm{B}} \mathrm{T}}\right)$. Inserting those expressions in equ.(57) will result in a temperature dependent helical pitch. It is even a priori possible to obtain a reversal of the helix direction when temperature varies.

\section{Conclusion}

By developing the dynamic interactions between molecules beyond the electric dipole approximation and by examining the symmetry properties of the resulting interaction energy terms, we have evidenced a term which exists only in optically active molecules and which can account for the helical configuration of chiral nematics. That term couples the quadrupole parts of the molecular optical activities and adds itself to the anisotropic Van der Waals interaction which couples the quadrupole parts of the molecular polarisabilities. The two couplings have the same behaviour with respect to rotations but different ranges, the latter is attractive and the former is repulsive. Their combination may generate a helical organization with a temperature independent pitch. Taking into account both the anisotropic part of the short range exclusion forces and the thermally induced effect of the static interactions can explain the actual temperature dependence of the pitch. No assumption has been made upon the shape of the molecules.

\section{Acknowledgement}

The author expresses its gratitude to Daniel Chemla (1940-2008) and wishes to pay a tribute to his memory. 
From the expression of the potential vector of the quantized electromagnetic field in the Coulomb gauge, i.e.

$$
\mathbf{A}=\sqrt{\frac{\hbar}{2 \epsilon_{0} \omega \mathrm{V}}} \sum_{\mathbf{e} \perp \mathbf{k}} \mathbf{e}\left(\mathrm{a} \exp [\mathrm{ik} \cdot \mathbf{r}-\mathrm{i} \omega \mathrm{t}]+\mathrm{a}^{+} \exp [\mathbf{i k} \cdot \mathbf{r}-\mathrm{i} \omega \mathrm{t}]\right)
$$

the transverse electric and magnetic fields are derived as

$$
\begin{aligned}
& \mathbf{E}_{\perp}=-\frac{\partial \mathbf{A}}{\partial \mathrm{t}}=\sqrt{\frac{\hbar \omega}{2 \epsilon_{0} V}} \sum_{\mathbf{e} \perp \mathbf{k}} \mathrm{ie}\left(\mathrm{a} \exp [\mathrm{ik} \cdot \mathbf{r}-\mathrm{i} \omega \mathrm{t}]-\mathrm{a}^{+} \exp [\mathrm{ik} \cdot \mathbf{r}-\mathrm{i} \omega \mathrm{t}]\right) \\
& \mathbf{B}=\boldsymbol{\nabla} \times \mathbf{A}=\sqrt{\frac{\hbar \omega}{2 \epsilon_{0} \mathrm{~V}} \sum_{\mathbf{e} \perp \mathbf{k}} \frac{\mathrm{i} \mathbf{k} \times \mathbf{e}}{\omega}\left(\mathrm{a} \exp [\mathrm{ik} \cdot \mathbf{r}-\mathrm{i} \omega \mathrm{t}]-\mathrm{a}^{+} \exp [\mathrm{ik} \cdot \mathbf{r}-\mathrm{i} \omega \mathrm{t}]\right)}
\end{aligned}
$$

The Green function associated to the propagation equation of the electromagnetic field is

$$
G_{\phi}\left(\omega^{2}\right)=\lim _{\varepsilon \rightarrow 0} \frac{1}{\omega^{2}-c^{2} k^{2}+i \varepsilon} .
$$

The Green function associated to the Schrödinger equation for the molecule A is

$$
G_{\mathrm{A}}(\mathrm{E})=\lim _{\varepsilon_{\mathrm{A}} \rightarrow 0} \frac{1}{\mathrm{E}-\mathcal{H}_{\mathrm{A}}+\mathrm{i} \varepsilon_{\mathrm{A}}}
$$

where $\mathcal{H}_{\mathrm{A}}$ is the molecular Hamiltonian $\mathcal{H}$ for $\mathrm{A}$.

The coupling hamiltonian of a molecular system with the transverse electromagnetic field can be expanded in the neighbourhood of the centre of the molecular charge distribution. At the lowest order, it evidences the electric dipole interaction (E1)

$$
\mathrm{V}^{(\mathrm{E} 1)}=-\mathbf{d} \cdot \mathbf{E}_{\perp}(\mathbf{R}) \text {, with } \mathbf{d}=\sum \mathrm{q} \mathbf{r} .
$$

The second order evidences the magnetic dipole term (M1) and the electric quadrupole term (E2), respectively being the anti-symmetric and symmetric contribution and being written as

$$
\mathrm{V}^{(\mathrm{M} 1)}=-\mathbf{M} \cdot \mathbf{B}(\mathbf{R}), \mathrm{V}^{(\mathrm{E} 2)}=-\frac{1}{2} \nabla \mathbf{E}_{\perp}(\mathbf{R}): \mathbf{Q}
$$

The third order accounts for the magnetic quadrupole (M2) and for the electric octupole (E3):

$$
\mathrm{V}^{(\mathrm{M} 2)}=-\frac{1}{2} \nabla \mathbf{B}(\mathbf{R}): \mathbf{M}^{(2)}, \mathrm{V}^{(\mathrm{E} 3)}=-\frac{1}{3 !} \nabla \nabla \mathbf{E}_{\perp}(\mathbf{R}): \mathbf{Q}^{(3)}
$$

After integrating the transition amplitude associated to diagram (i) of fig. (1) over the 4 interaction times between an initial instant $t_{i} \rightarrow-\infty$ when the field - matter coupling is switched on and a final instant $t_{f} \rightarrow+\infty$ where it is switched off, it can be expressed as 


$$
\begin{aligned}
& \mathrm{T}_{\mathrm{i} \rightarrow \mathrm{f}}^{\prime}=\lim _{\varepsilon, \varepsilon^{\prime} \rightarrow 0}(2 \pi)^{-1}\left(\mathrm{t}_{\mathrm{f}}-\mathrm{t}_{\mathrm{i}}\right) \exp \left[-\mathrm{iE}_{0}\left(\mathrm{t}_{\mathrm{f}}-\mathrm{t}_{\mathrm{i}}\right) \hbar^{-1}\right] \int_{-\infty}^{+\infty} \omega^{4} \mathrm{~d} \omega
\end{aligned}
$$

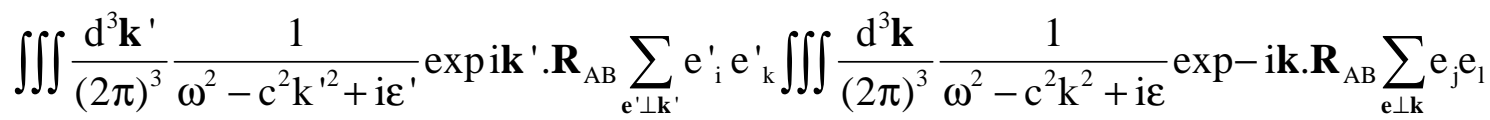

$$
\begin{aligned}
& \epsilon_{0}^{-1} \sum_{n} \frac{\left\langle 0\left|d_{i}(B) P_{n} d_{j}(B)\right| 0\right\rangle}{E_{0}-E_{n}+\hbar \omega-\Delta_{n}(\omega)+\frac{i \Gamma_{n}(\omega)}{2}} \epsilon_{0}^{-1} \sum_{m} \frac{\left\langle 0\left|d_{k}(A) P_{m} d_{1}(A)\right| 0\right\rangle}{E_{0}-E_{m}-\hbar \omega-\Delta_{m}(-\omega)-\frac{i \Gamma_{m}(-\omega)}{2}}
\end{aligned}
$$

In a similar way, the transition amplitude associated to diagram (ii) of fig. (1) is

$$
\begin{aligned}
& \mathrm{T}_{\mathrm{i} \rightarrow \mathrm{f}}^{\prime}=\lim _{\varepsilon, \varepsilon^{\prime}, \varepsilon_{\mathrm{A}}, \varepsilon_{\mathrm{B}} \rightarrow 0}(2 \pi)^{-1}\left(\mathrm{t}_{\mathrm{f}}-\mathrm{t}_{\mathrm{i}}\right) \exp \left[-\mathrm{iE}_{0}\left(\mathrm{t}_{\mathrm{f}}-\mathrm{t}_{\mathrm{i}}\right) \hbar^{-1}\right] \int_{-\infty}^{+\infty} \omega^{4} \mathrm{~d} \omega
\end{aligned}
$$

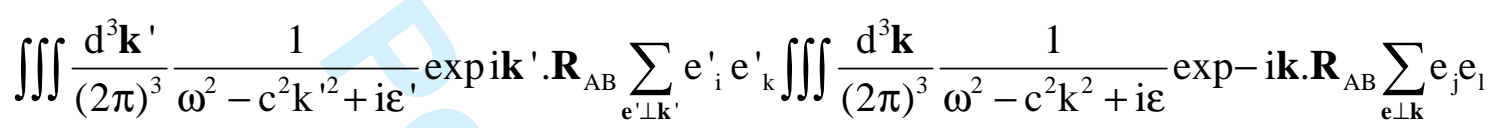

$$
\begin{aligned}
& \epsilon_{0}^{-1} \sum_{n} \frac{\left\langle 0\left|d_{j}(B) P_{n} d_{i}(B)\right| 0\right\rangle}{E_{0}-E_{n}-\hbar \omega-\Delta_{n}(-\omega)-\frac{i \Gamma_{n}(-\omega)}{2}} \epsilon_{0}^{-1} \sum_{m} \frac{\left\langle 0\left|d_{k}(A) P_{m} d_{1}(A)\right| 0\right\rangle}{E_{0}-E_{m}-\hbar \omega-\Delta_{m}(-\omega)-\frac{i \Gamma_{m}(-\omega)}{2}}
\end{aligned}
$$

The total transition amplitude is $\mathrm{T}_{\mathrm{i} \rightarrow \mathrm{f}}=\mathrm{T}_{\mathrm{i} \rightarrow \mathrm{f}}^{0}+\mathrm{T}_{\mathrm{i} \rightarrow \mathrm{f}}^{\prime}+\mathrm{T}^{\prime \prime}{ }_{\mathrm{i} \rightarrow \mathrm{f}}$ where $\mathrm{T}_{\mathrm{i} \rightarrow \mathrm{f}}^{0}=\exp \left[-\mathrm{iE}_{0}\left(\mathrm{t}_{\mathrm{f}}-\mathrm{t}_{\mathrm{i}}\right) \hbar^{-1}\right]$ accounts for the trivial non perturbated case.

That expression of $\mathrm{T}_{\mathrm{i} \rightarrow \mathrm{f}}$ can be written in a more convenient way by restricting the variation range of $\omega$ to $\omega>0$. If then we introduce the molecular polarisability tensor

$$
\alpha_{\mathrm{kl}}(\omega)=\left(4 \pi \epsilon_{0}\right)^{-1} \sum_{\mathrm{m}} \frac{\left\langle 0\left|\mathrm{~d}_{\mathrm{k}} \mathrm{P}_{\mathrm{m}} \mathrm{d}_{1}\right| 0\right\rangle}{\mathrm{E}_{0}-\mathrm{E}_{\mathrm{m}}+\hbar \omega-\Delta_{\mathrm{m}}(\omega)+\frac{\mathrm{i} \Gamma_{\mathrm{m}}(\omega)}{2}}+\frac{\left\langle 0\left|\mathrm{~d}_{\mathrm{l}} \mathrm{P}_{\mathrm{m}} \mathrm{d}_{\mathrm{k}}\right| 0\right\rangle}{\mathrm{E}_{0}-\mathrm{E}_{\mathrm{m}}-\hbar \omega-\Delta_{\mathrm{m}}(-\omega)-\frac{\mathrm{i} \Gamma_{\mathrm{m}}(-\omega)}{2}}
$$

and if we use the symmetry property $\alpha_{\mathrm{kl}}(-\omega)=\alpha_{\mathrm{lk}} *(\omega), \mathrm{T}_{\mathrm{i} \rightarrow \mathrm{f}}$ becomes

$$
\begin{aligned}
\mathrm{T}_{\mathrm{i} \rightarrow \mathrm{f}}= & \mathrm{T}_{\mathrm{i} \rightarrow \mathrm{f}}^{0}+\lim _{\varepsilon, \varepsilon^{\prime} \rightarrow 0}(2 \pi)^{-1}\left(\mathrm{t}_{\mathrm{f}}-\mathrm{t}_{\mathrm{i}}\right) \exp \left[-\mathrm{iE}_{0}\left(\mathrm{t}_{\mathrm{f}}-\mathrm{t}_{\mathrm{i}}\right) \hbar^{-1}\right] \int_{0}^{+\infty} \mathrm{d} \omega \sum_{\mathrm{ijkl}} \alpha_{\mathrm{ij}}(\omega)_{\mathrm{B}} \alpha_{\mathrm{kl}}(\omega)_{\mathrm{A}} \\
& \left(4 \pi \omega^{2}\right) \iiint_{\frac{\mathrm{d}^{3} \mathbf{k}^{\prime}}{(2 \pi)^{3}} \frac{1}{\omega^{2}-\mathrm{c}^{2} \mathrm{k}^{\prime 2}+\mathrm{i} \varepsilon^{\prime}}} \exp \mathbf{k k}^{\prime} \cdot \mathbf{R}_{\mathrm{AB}} \sum_{\mathbf{e}^{\prime} \perp \mathbf{k}^{\prime}} \mathrm{e}_{\mathrm{i}}^{\prime} \mathrm{e}^{\prime}{ }_{\mathrm{k}} \\
& \left(4 \pi \omega^{2}\right) \iiint \frac{\mathrm{d}^{3} \mathbf{k}}{(2 \pi)^{3}} \frac{1}{\omega^{2}-\mathrm{c}^{2} \mathrm{k}^{2}+\mathrm{i} \varepsilon} \exp -\mathrm{ik} \cdot \mathbf{R}_{\mathrm{AB}} \sum_{\mathbf{e} \perp \mathbf{k}} \mathrm{e}_{\mathrm{j}} \mathrm{e}_{1}
\end{aligned}
$$

That expression holds even if the polarisability is not purely real. Also note that $\gamma(\omega) \propto \omega^{3}$. We now have to explicit 
$\iiint \frac{\mathrm{d}^{3} \mathbf{k}}{(2 \pi)^{3}} \frac{1}{\omega^{2}-\mathrm{c}^{2} \mathrm{k}^{2}+\mathrm{i} \varepsilon} \exp -\mathrm{ik} \cdot \mathbf{R}_{\mathrm{AB}} \sum_{\mathbf{e} \perp \mathbf{k}} \mathrm{e}_{\mathrm{j}} \mathrm{e}_{1}$.

It is easy to check that $\sum_{\mathbf{e} \perp \mathbf{k}} \mathrm{e}_{\mathrm{j}} \mathrm{e}_{1}=\delta_{\mathrm{jl}}-\frac{\mathrm{k}_{\mathrm{j}} \mathrm{k}_{1}}{\mathrm{k}^{2}}$.

Then we have to calculate the two integrals

$$
\lim _{\varepsilon \rightarrow 0} \iiint \frac{d^{3} \mathbf{k}}{(2 \pi)^{3}} \frac{1}{\omega^{2}-c^{2} k^{2}+i \varepsilon} \exp -i \mathbf{k} \cdot \mathbf{R}_{A B}
$$

and

$$
\lim _{\varepsilon \rightarrow 0} \iiint \frac{\mathrm{d}^{3} \mathbf{k}}{(2 \pi)^{3}} \frac{\mathrm{k}_{\mathrm{j}} \mathrm{k}_{1}}{\mathrm{k}^{2}} \frac{1}{\omega^{2}-\mathrm{c}^{2} \mathrm{k}^{2}+\mathrm{i} \varepsilon} \exp -\mathrm{ik} \cdot \mathbf{R}_{\mathrm{AB}}
$$

The second integral is

$$
\lim _{\varepsilon \rightarrow 0}-\frac{c^{2}}{\omega^{2}} \nabla_{j} \nabla_{1} \iiint \frac{d^{3} \mathbf{k}}{(2 \pi)^{3}} \frac{1}{\omega^{2}-c^{2} k^{2}+i \varepsilon} \exp -i k \cdot R_{A B}
$$

and the first integral is

$$
\lim _{\varepsilon \rightarrow 0} \frac{4 \pi}{(2 \pi)^{3}} \int_{-\infty}^{+\infty} \frac{k^{2} d k}{\omega^{2}-c^{2} k^{2}+i \varepsilon} \frac{\operatorname{expikR}}{2 i k R}=-\frac{\operatorname{expi\omega R} / c}{4 \pi c^{2} R}
$$

We finally obtain

$$
\lim _{\varepsilon \rightarrow 0} \iiint \frac{\mathrm{d}^{3} \mathbf{k}}{(2 \pi)^{3}} \frac{1}{\omega^{2}-\mathrm{c}^{2} \mathrm{k}^{2}+\mathrm{i} \varepsilon} \exp -\mathrm{ik} \cdot \mathbf{R}_{\mathrm{AB}} \sum_{\mathbf{e} \perp \mathbf{k}} \mathrm{e}_{\mathrm{j}} \mathrm{e}_{1}=-\left(\delta_{\mathrm{j} 1}+\frac{\mathrm{c}^{2}}{\omega^{2}} \nabla_{\mathrm{j}} \nabla_{1}\right)\left(\frac{\operatorname{expi\omega R} / \mathrm{c}}{4 \pi \mathrm{c}^{2} \mathrm{R}}\right)
$$

In a similar way we obtain

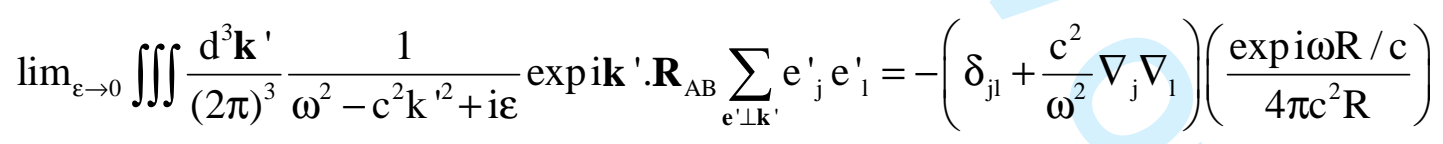

It is then a straightforward matter to derive

$$
\begin{aligned}
& \nabla_{\mathrm{j}} \frac{\operatorname{expi\omega R} / \mathrm{c}}{\mathrm{R}}=\frac{\mathrm{R}_{\mathrm{j}}}{\mathrm{R}^{3}}\left(\frac{\mathrm{i} \omega \mathrm{R}}{\mathrm{c}}-1\right) \operatorname{expi\omega R} / \mathrm{c} \\
& \nabla_{\mathrm{j}} \nabla_{1} \frac{\operatorname{expi\omega R} / \mathrm{c}}{\mathrm{R}}=\frac{\mathrm{R}_{\mathrm{j}} \mathrm{R}_{1}}{\mathrm{R}^{5}}\left(\left(\frac{\mathrm{i} \omega \mathrm{R}}{\mathrm{c}}\right)^{2}-3\left(\frac{\mathrm{i} \omega \mathrm{R}}{\mathrm{c}}\right)+3\right) \operatorname{expi\omega R} / \mathrm{c}+\frac{\delta_{\mathrm{jl}}}{\mathrm{R}^{3}}\left(\frac{\mathrm{i} \omega \mathrm{R}}{\mathrm{c}}-1\right) \operatorname{expi\omega R} / \mathrm{c}
\end{aligned}
$$




\section{APPENDIX A2}

Any 3-dimension Cartesian tensor can be split in a unique way into parts irreducible simultaneously with respect to the rotation group and to the index permutation group [28]. The symmetry of an irreducible Cartesian tensor will then be unambiguously characterized by the labels of the irreducible representations of both groups. Let $\{\Gamma\} \mathrm{T}^{(\mathrm{J})}{ }_{\mathrm{ijk} \ldots . .1}$ designate an irreducible component of the Cartesian tensor $\mathrm{T}_{\mathrm{ijk} . . .1}$ belonging to the irreducible representation $\{\Gamma\}$ of the index permutation group and to the irreducible representation of weight $\mathbf{J}$ of the rotation group.

$D_{j 1}(R)$ as given by equ.(4) has two irreducible components $\{2\} D^{(0)}{ }_{j 1}$ and $\{2\} D^{(2)}{ }_{j 1}$.

From its definition equ. (20), $\mathrm{F}_{\mathrm{jlq}}(\mathrm{R})$ is proportional to $\nabla_{\mathrm{q}} \frac{1}{\mathrm{R}^{3}} \mathrm{D}_{\mathrm{jl}}(\mathrm{R})$ and it can be checked that it has three irreducible components $\{3\} \mathrm{F}^{(1)}{ }_{\mathrm{jlq}},\{3\} \mathrm{F}^{(3)}{ }_{\mathrm{jlq}}$ and $\{21\} \mathrm{F}^{(1)}{ }_{\mathrm{jlq}}$. Similarly from its definition equ.(28), $\mathrm{G}_{\mathrm{jlpq}}(\mathrm{R})$ is proportional to $\nabla_{\mathrm{p}} \nabla_{\mathrm{q}} \frac{1}{\mathrm{R}^{3}} \mathrm{D}_{\mathrm{jl}}(\mathrm{R})$ and it can be checked that it has four irreducible components $\{4\} \mathrm{G}^{(0)}{ }_{\mathrm{jlpq}},\{4\} \mathrm{G}_{\mathrm{jlpq}}^{(2)},\{4\} \mathrm{G}_{\mathrm{jlpq}}^{(4)},\left\{2^{2}\right\} \mathrm{G}^{(0)}{ }_{\mathrm{jlpq}}$ and $\left\{2^{2}\right\} \mathrm{G}^{(2)}{ }_{\mathrm{jlpq}}$.

The scalar product of two Cartesian tensors $\mathrm{T}_{\mathrm{pq} . . .}^{1}$ and $\mathrm{T}_{\mathrm{pq} . . .}^{2}$ of same rank is defined by $\mathrm{T}^{1} . \mathrm{T}^{2}=\sum_{\mathrm{pq} \ldots} \mathrm{T}_{\mathrm{pq} \ldots}^{1} \mathrm{~T}_{\mathrm{pq} \ldots .}^{2}$ and the norm $\left\|\mathrm{T}_{\mathrm{pq} . . .}\right\|$ of $\mathrm{T}_{\mathrm{pq} \ldots}$ by $\left\|\mathrm{T}_{\mathrm{pq} \ldots}\right\|^{2}=\sum_{\mathrm{pq} \ldots} \mathrm{T}_{\mathrm{pq} \ldots} \mathrm{T}_{\mathrm{pq} \ldots}$. The following Cartesian tensors are fully symmetric with respect to index permutations and their norm is equal to 1:

$$
\begin{aligned}
& \mathfrak{S}_{\mathrm{jl}}=\frac{\delta_{\mathrm{jl}}}{\sqrt{3}} \\
& \mathfrak{D}_{\mathrm{jl}}=\frac{3 \mathrm{R}_{\mathrm{j}} \mathrm{R}_{1}-\mathrm{R}^{2} \delta_{\mathrm{jl}}}{\sqrt{6} \mathrm{R}^{2}} \\
& \mathfrak{F}_{\mathrm{jlq}}=\frac{5 \mathrm{R}_{\mathrm{j}} \mathrm{R}_{1} \mathrm{R}_{\mathrm{q}}-\mathrm{R}_{\mathrm{j}} \mathrm{R}^{2} \delta_{\mathrm{lq}}-\mathrm{R}_{1} \mathrm{R}^{2} \delta_{\mathrm{jq}}-\mathrm{R}_{\mathrm{q}} \mathrm{R}^{2} \delta_{\mathrm{jl}}}{2 \mathrm{R}^{3}} \\
& \mathfrak{G}_{\mathrm{jlpq}}=\frac{35 \mathrm{R}_{\mathrm{j}} \mathrm{R}_{\mathrm{l}} \mathrm{R}_{\mathrm{p}} \mathrm{R}_{\mathrm{q}}-5 \mathrm{R}_{\mathrm{j}} \mathrm{R}_{\mathrm{q}} \mathrm{R}^{2} \delta_{\mathrm{pl}}-5 \mathrm{R}_{\mathrm{j}} \mathrm{R}_{\mathrm{p}} \mathrm{R}^{2} \delta_{\mathrm{q} 1}-5 \mathrm{R}_{\mathrm{j}} \mathrm{R}_{\mathrm{l}} \mathrm{R}^{2} \delta_{\mathrm{pq}}-5 \mathrm{R}_{\mathrm{p}} \mathrm{R}_{\mathrm{q}} \mathrm{R}^{2} \delta_{\mathrm{jl}}}{-5 \mathrm{R}_{\mathrm{p}} \mathrm{R}_{\mathrm{l}} \mathrm{R}^{2} \delta_{\mathrm{jq}}-5 \mathrm{R}_{\mathrm{q}} \mathrm{R}_{\mathrm{l}} \mathrm{R}^{2} \delta_{\mathrm{jp}}+\mathrm{R}^{4} \delta_{\mathrm{jl}} \delta_{\mathrm{pq}}+\mathrm{R}^{4} \delta_{\mathrm{jp}} \delta_{\mathrm{lq}}+\mathrm{R}^{4} \delta_{\mathrm{jq}} \delta_{\mathrm{lp}}} \\
& 8 \mathrm{R}^{4}
\end{aligned}
$$

In the short range limit,

$$
\begin{aligned}
& \{2\} \mathrm{D}^{(0)}{ }_{\mathrm{j} 1} \rightarrow 0,\{2\} \mathrm{D}^{(2)}{ }_{\mathrm{jl}} \rightarrow \frac{-3 \mathrm{R}_{\mathrm{j}} \mathrm{R}_{1}+\mathrm{R}^{2} \delta_{\mathrm{j} 1}}{\mathrm{R}^{2}}=-\sqrt{6} \mathfrak{D}_{\mathrm{jl}}, \\
& \{3\} \mathrm{F}^{(1)}{ }_{\mathrm{jlq}} \rightarrow 0,\{21\} \mathrm{F}^{(1)}{ }_{\mathrm{jlq}} \rightarrow 0,
\end{aligned}
$$




$$
\begin{aligned}
& \{3\} \mathrm{F}^{(3)}{ }_{\mathrm{jlq}} \rightarrow \nabla_{\mathrm{q}}\left(\frac{-3 \mathrm{R}_{\mathrm{j}} \mathrm{R}_{1}+\mathrm{R}^{2} \delta_{\mathrm{j} 1}}{\mathrm{R}^{5}}\right)=\frac{6}{\mathrm{R}^{4}} \mathfrak{F}_{\mathrm{jlq}}, \\
& \{4\} \mathrm{G}^{(0)}{ }_{\mathrm{j} \text { lpq }} \rightarrow 0,\{4\} \mathrm{G}^{(2)}{ }_{\mathrm{jlpq}} \rightarrow 0,\left\{2^{2}\right\} \mathrm{G}^{(0)}{ }_{\mathrm{jlpq}} \rightarrow 0,\left\{2^{2}\right\} \mathrm{G}^{(2)}{ }_{\mathrm{jlpq}} \rightarrow 0, \\
& \{4\} \mathrm{G}^{(4)}{ }_{\mathrm{j} \text { lpq }} \rightarrow-\nabla_{\mathrm{p}} \nabla_{\mathrm{q}}\left(\frac{-3 \mathrm{R}_{\mathrm{j}} \mathrm{R}_{1}+\mathrm{R}^{2} \delta_{\mathrm{jl}}}{\mathrm{R}^{5}}\right)=\frac{24}{\mathrm{R}^{5}} \mathfrak{G}_{\mathrm{jlpq}}
\end{aligned}
$$




\section{APPENDIX A3}

The tensor scalar product of two spherical tensors $\mathrm{T}^{(\mathrm{K})}$ and $\mathrm{T}^{\prime(\mathrm{K})}$ of same weight $\mathrm{K}$ is defined by

$$
\begin{aligned}
& \left(\mathrm{T}^{(\mathrm{K})} \cdot \mathrm{T}^{(\mathrm{K})}\right)=\sum_{\mathrm{Q}=-\mathrm{K}}^{+\mathrm{K}}(-1)^{\mathrm{Q}} \mathrm{T}_{\mathrm{Q}}^{(\mathrm{K})} \mathrm{T}_{-\mathrm{Q}}^{\prime(\mathrm{K})} \\
& \text { and the norm }\left\|\mathrm{T}^{(\mathrm{K})}\right\| \text { of } \mathrm{T}^{(\mathrm{K})} \text { by } \\
& \left\|\mathrm{T}^{(\mathrm{K})}\right\|^{2}=\left(\mathrm{T}^{(\mathrm{K})} \cdot \mathrm{T}^{(\mathrm{K})}\right)=\sum_{\mathrm{Q}=-\mathrm{K}}^{+\mathrm{K}}(-1)^{\mathrm{Q}} \mathrm{T}_{\mathrm{Q}}^{(\mathrm{K})} \mathrm{T}_{-\mathrm{Q}}^{(\mathrm{K})}
\end{aligned}
$$

The irreducible spherical unit tensors $\mathrm{C}_{\mathrm{q}}^{(\mathrm{K})}(\theta, \varphi)$ 's are connected to the usual orthonormal spherical harmonic functions by [29]

$$
\mathrm{C}_{\mathrm{q}}^{(\mathrm{K})}(\theta, \varphi)=\sqrt{\frac{4 \pi}{2 \mathrm{~K}+1}} \mathrm{Y}_{\mathrm{KQ}}(\theta, \varphi)
$$

One has

$$
\mathrm{C}_{\mathrm{Q}}^{(\mathrm{K}) *}(\theta, \varphi)=(-1)^{\mathrm{Q}} \mathrm{C}_{-\mathrm{Q}}^{(\mathrm{K})}(\theta, \varphi)
$$

and

$$
\int \frac{\mathrm{d} \Omega}{4 \pi}(-1)^{\mathrm{Q}} \mathrm{C}_{-\mathrm{Q}}^{(\mathrm{K})} \mathrm{C}_{\mathrm{Q}^{\prime}}^{\left(\mathrm{K}^{\prime}\right)}=\frac{\delta_{\mathrm{KK}} \delta_{\mathrm{QQ}^{\prime}}}{2 \mathrm{~K}+1}
$$

$C_{0}^{(K)}(\theta, \varphi)$ is $P_{K}(\cos \theta)$, i.e. the Legendre polynomial of order $K$.

The scalar product $\left(\mathrm{C}^{(\mathrm{K})}\left(\theta_{\mathrm{A}}, \varphi_{\mathrm{A}}\right) \cdot \mathrm{C}^{(\mathrm{K})}\left(\theta_{\mathrm{B}}, \varphi_{\mathrm{B}}\right)\right)$ simply is $\mathrm{P}_{\mathrm{K}}\left(\cos \theta_{\mathrm{AB}}\right)$ with $\cos \theta_{\mathrm{AB}}=\cos \theta_{\mathrm{A}} \cos \theta_{\mathrm{B}}+\sin \theta_{\mathrm{A}} \sin \theta_{\mathrm{B}} \cos \left(\varphi_{\mathrm{A}}-\varphi_{\mathrm{B}}\right)$ whereas $\left\|\mathrm{C}^{(\mathrm{K})}\right\|=1$. 


\section{Figure caption:}

Fig. 1: The two types of dynamic interaction diagrams between 2 molecules via the exchange of two photons.

Fig. 2: Relative configuration of the molecular reference frames: Case of intersecting molecular planes.

Fig. 3: Relative configuration of the molecular reference frames: Case of parallel molecular planes. 
Fig. 1

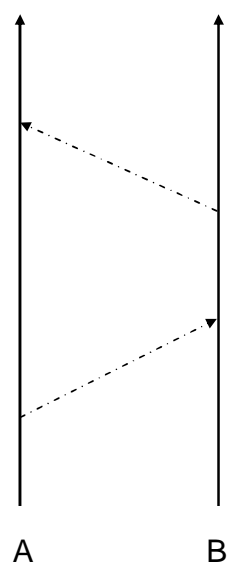

(i)

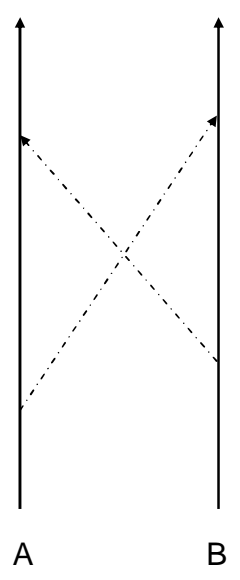

(ii) 
Fig. 2

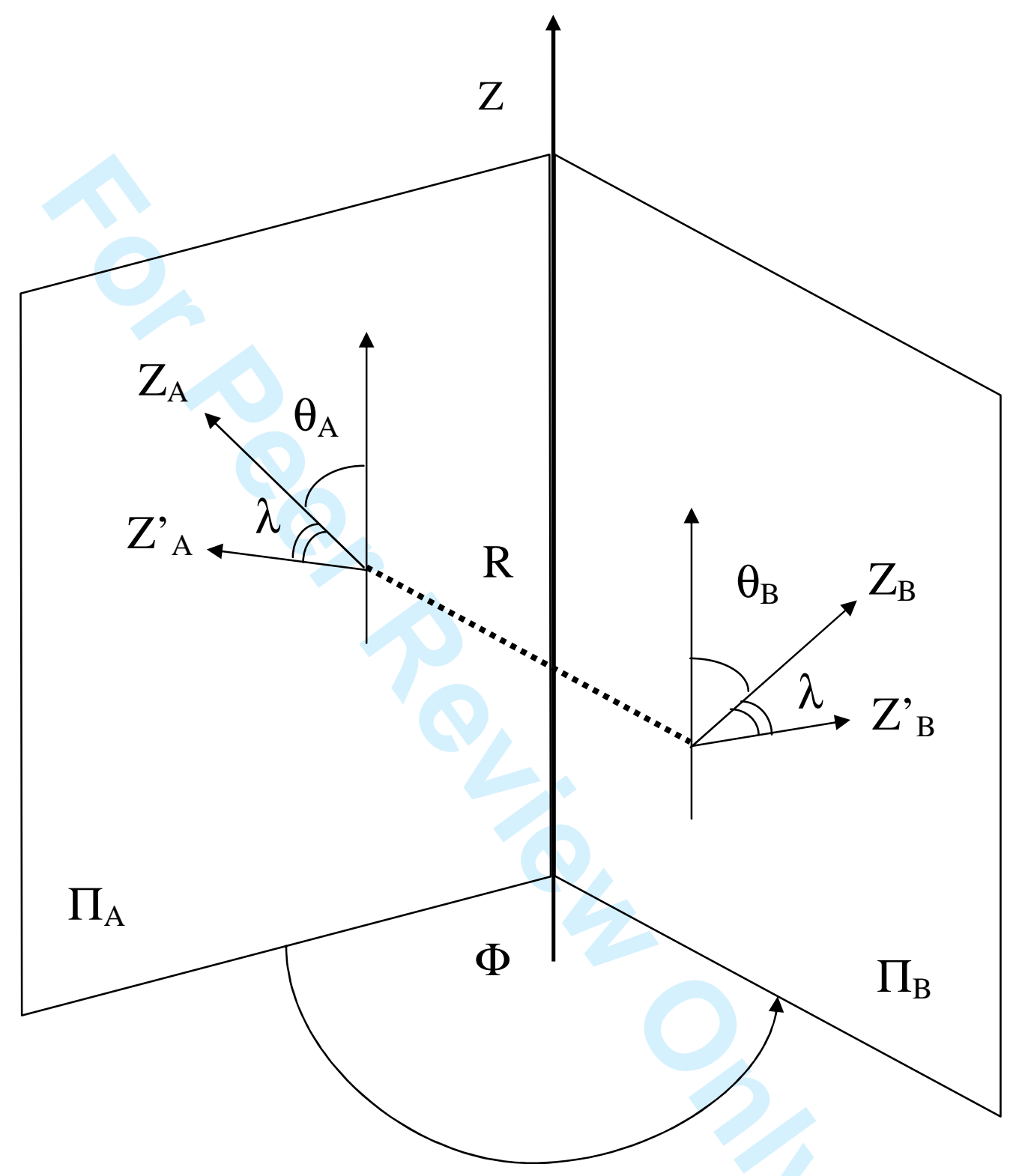


Fig. 3

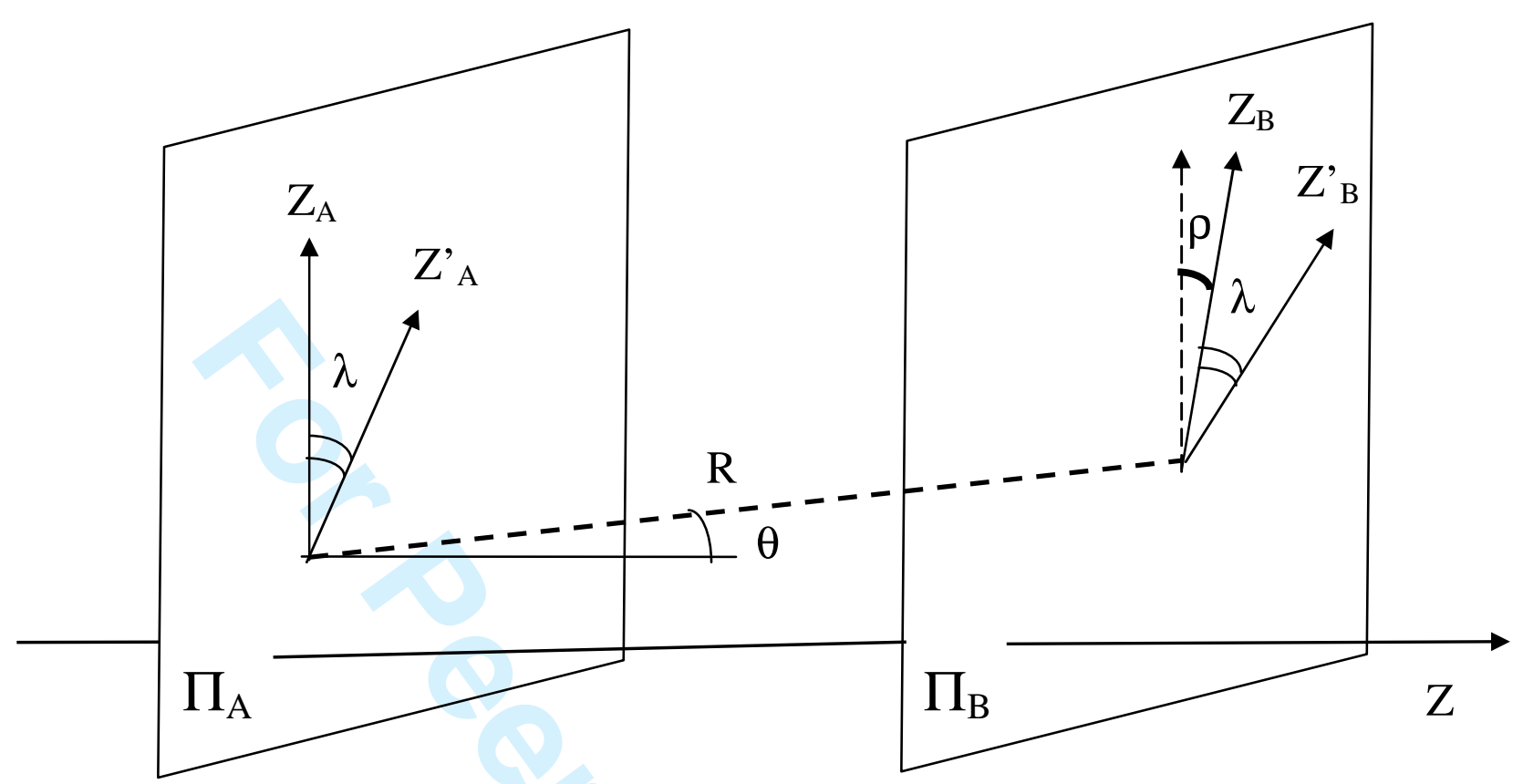


[1] H.B.G. Casimir, D. Polder, Phys. Rev. 73, 4, 360 (1948)

[2] J.K. Jenkins, A. Salam, T. Thirunamachandran, Phys. Rev. A 50, 4767 (1994)

[3] J.K. Jenkins, A. Salam, T. Thirunamachandran, Mol. Phys. 82, 835 (1995)

[4] E.A. Power, T. Thirunamachandran, Phys. Rev. A 51, 3660 (1995)

[5] A. Salam, T. Thirunamachandran, J. Chem. Phys. 104, 5094 (1996)

[6] R Passante, E.A. Power, T Thirunamachandran, Phys. Lett. A, 249 (1998)

[7] E.A. Power, T. Thirunamachandran, Phys. Rev. A 60, 4927 (1999)

[8] C. Cohen-Tannoudji, J. Dupont-Roc, G. Grynberg, Photons et atomes, introduction à l'électrodynamique quantique (InterEditions \& Editions du C.N.R.S., Paris, 1987). English version: Photons and atoms, introduction to quantum electrodynamics (Wiley, New-York, 1989)

[9] C. Cohen-Tannoudji, J. Dupont-Roc, G. Grynberg, Processus d'interaction entre photons et atomes (InterEditions \& Editions du C.N.R.S., Paris, 1988). English version: Atom-photon interactions : basic processes and applications (Wiley, New-York, 1992)

[10] T. Thirunamachandran, Phys. Scr. T 21, 123 (1988)

[11] D.P. Craig, T. Thirunamachandran, Molecular Quantum Electrodynamics (Dover NY 1998)

[12] R. Loudon, The quantum theory of light (Clarendon Press, Oxford, 1973)

[13] L. Landau, E. Lifshitz, Electrodynamics of continuous media (MIR, Moscow, 1969)

[14] P.G. de Gennes, J. Prost, The Physics of Liquid Crystals, (Clarendon Press, Oxford, 1993)

[15] R.A. Harris, Mol. Phys. 105, 2433 (2007)

[16] P.E. Schipper, Chem. Phys. 26, 29 (1977)

[17] D.P. Craig, in Optical activity and chiral discrimination, ed. S.F. Mason (Reidel, NewYork, 1979)

[18] D.P. Craig, P.E. Schipper, Proc. R. Soc. A 342, 19 (1974)

[19] W. Maier and A. Saupe, Z. Naturforsch. 13 a, 564 (1958)

[20] G. Vertogen, B.W. van der Meer, Phys. Rev. A 19, 370 (1979)

[21] W.J.A. Goossens, Mol. Cryst. Liquid Crystals 12, 237 (1970)

[22] B.W. van der Meer, G. Vertogen, A.J. Dekker, J.G.J. Ypma, J. Chem. Phys. 65, 3935 (1976)

[23] A.B. Harris, D.K. Kamien, T.C. Lubensky, Rev. Mod. Phys. 71, 1745 (1999)

[24] Y.R. Lin-Liu, Y.M. Shih, C.W. Woo, H.T. Tan, Phys. Rev. A 14, 445 (1976)

[25] A.B. Harris, R. Kamien, T.C. Lubenski, Phys. Rev. Let. 78, 1476 (1997)

[26] S.A. Issaenko, A.B. Harris, T.C. Lubenski, Phys. Rev. E 60, 578 (1999) 
[27] S.A. Issaenko, A.B. Harris, Phys. Rev. E 61, 2777 (2000)

[28] M. Hammermesh, Group theory and its application to physical problems (AddisonWesley, New-York, 1964)

[29] A.R. Edmonds, Angular momentum in quantum mechanics (Princeton University Press, Princeton, 1960) 